\title{
Knowing Action Boundaries: \\ Understanding and Mitigating Over-Estimation Bias in Reaching
}

\author{
Rebecca Anne Tokay Weast \\ B.A., Franklin and Marshall College, 2009 \\ M.A., University of Virginia, 2014
}

\begin{abstract}
A Dissertation Presented to the Graduate Faculty of the University of Virginia in Candidacy for the Degree of Doctor of Philosophy
\end{abstract}

\section{Department of Psychology}

University of Virginia

April, 2016 


\begin{abstract}
The typical person can navigate their world day in and day out without making too many errors, and without giving such navigation much thought. Such continued success would indicate that people know, more or less, what they can do when presented with an environment. Several decades of research, however, have shown that this is not the case. When asked to estimate, for example, the extent of their arm's reach, individuals will typically over-estimate the extent of their reach by $10 \%$. While this figure fluctuates from between $5 \%$ to about $30 \%$ depending on the study, the presence of such an over-estimation bias is robust and consistent across the literature. The current work began by assessing some possible simple experimental causes of this over-estimation bias in reaching, and continued on to examine the extent to which this bias influences not just estimates of one's ability to act, but the actions themselves. Finally, the current work assessed to what degree participants could learn to be more accurate in both their actions and their judgments of their abilities.
\end{abstract}

A set of two studies found that over-estimation bias does not result from two typical aspects of the methods used in reaching experiments; participants are not driven to over-estimate their reach by the connotations of effortful extension contained within the word 'reach', nor were their biases apparently drive by the method by which they maintained their upright posture during the experiments. Over-estimation bias proves to be a real and robust phenomenon.

A subsequent set of three studies found that actions, while they show a slight bias to over-estimate, are not as biased as judgments. Further, the repetition within these experiments (detailed in Chapter 5) facilitated learning in participants: both participants' actions and judgments showed improved accuracy from the beginning to the end of their participation. The methods of these studies do not afford participants any visual, tactile, or external feedback about 
their performance, so, most interestingly, all of this improvement is apparently driven by proprioceptive feedback, coupled with the intermittent visual information of the target's location on the table. Taken together, these studies indicate that perceptions are consistently biased to a greater degree than actions, but both can improve with practice but without continuous visual feedback. 
Table of Contents

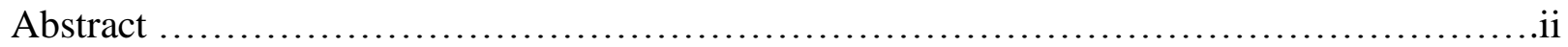

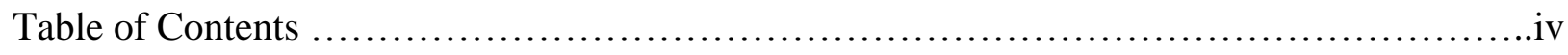

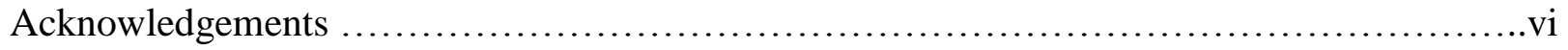

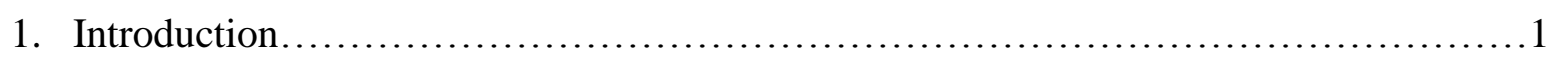

1.1 Defining Action Boundaries............................................

1.2 Action Boundaries Scale Perception of the Environment.........................

1.3 The Study of Action Boundaries: Typical Methods............................

1.4 Perceived and Actual Action Boundaries Differ: Three Accounts .................11

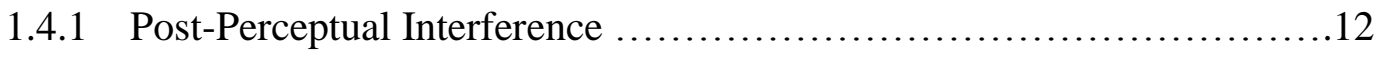

1.4.2 The Postural Stability Hypothesis ............................... 14

1.4.3 The Whole Body Engagement Hypothesis ..........................16

1.5 Learning Action Boundaries through experience and over the lifespan ............18

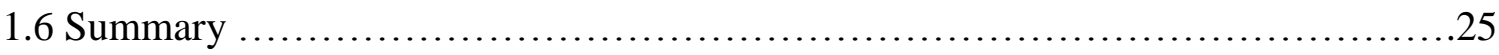

2. Study 1: Longer Reaction Times At and Around Action Boundaries....................27

3. Study Set 2: Over-Estimation Bias is Not an Artifact of Experimental Methods.........33

3.1 Study 2a: Active Posture Control Does Not Mitigate Over-Estimation .............34

3.2 Study 2b: The Semantics of Typical Instructions Do Not Drive Over-Estimation .38

4. Study Set 3: Learning is Inconsistent with Minimal Task-Specific Experience ........42

4.1 Study 3a: Task-Specific Experience Reduces Over-Estimation Bias without Vision

4.2 Study 3b: Experience is Not Sufficient to Reduce Over-Estimation ..............48 
4.3 Study 3c: Explicit Instructions Do Not Facilitate Reduction of Over-Estimation

5. Study Set 4: Actions are Less Biased Than Judgments, and Learning Occurs Without

Vision .55

5.1 Study 4a: Actions Show Less Bias and Learning Occurs in Both Actions and

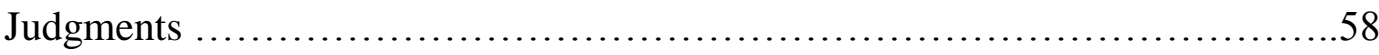

5.2 Study 4b: Actions Show Less Bias, but No Evidence of Learning ...............65

5.3 Study 4c: Actions Show Less Bias, Learning Occurs, and Awareness of Accuracy Does Not Drive Such Learning ...........................................69

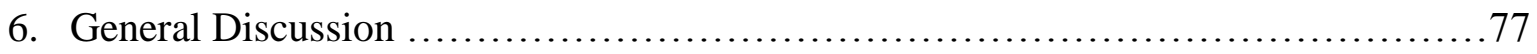

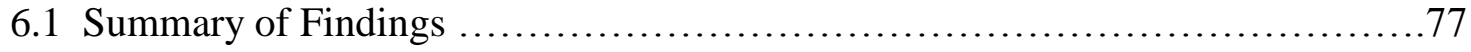

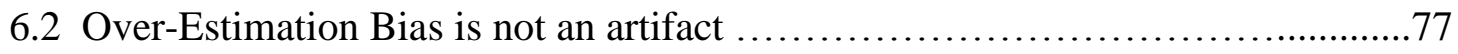

6.3 Actions are less biased than judgments.......................................

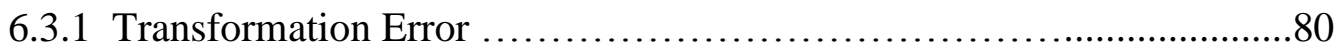

6.3.2 Two-Component Model of Target-Directed Actions ....................81

6.3.3 Dual-Route Model of Visual Perception .................................83

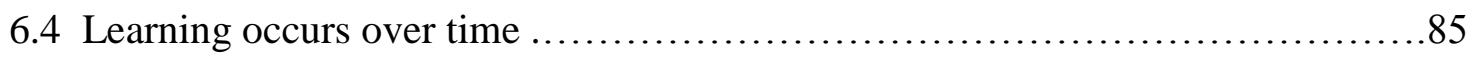

6.4.1 Learning Occurs Below the Level of Conscious Awareness ..................89

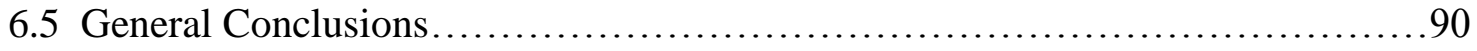




\section{Acknowledgements}

This work would not have been possible without the diligent and hard work of all of my undergraduate research assistants.

I have had the great fortune of sharing lab space and research ideas with so many outstanding fellow graduate students, including but certainly not limited to E. Blair Gross, Veronica Weser, Gianluca Finotti, Elyssa Twedt, and John Zadra. It has been a pleasure and a privilege developing ideas and creating new knowledge alongside each of you, growing intellectually and personally through knowing each of you and your unique approaches to work and life.

I don't know that my life would have taken this path after I completed my undergraduate work were it not for Fred Owens who, at Franklin and Marshall College, introduced me to the ecological approach to perception and fundamentally changed how I think about and experience the world.

Perhaps most of all, I am grateful for the hard work, guidance, wisdom and patience of my advisor, Denny Proffitt. Thank you for (among many, many other things) keeping me on track, teaching me how to ask better questions, and always reminding me of the correct tense in which to write $:$

I also must note my wonderful boyfriend Stanley Beckwith who has given me perspective and support throughout much of the work discussed in the pages to follow.

Finally, I would like to acknowledge my parents and my brother who always expected the best of me, and somehow put up with me through my awkward teenage years. 


\section{Introduction}

Actions influence perception, just as perception guides action. Several decades of research have established that the environment is not perceived in universal, arbitrary units, but instead that perceptions are scaled by the perceiver's ability to act on the environment. All observers have differently shaped/sized bodies of varying levels of strength and skill, which allow them to interact with the world in different ways. Because of this, observers experience perceptual worlds that have been uniquely scaled to their action capabilities. People are consistent and relatively accurate when judging their capacity for various kinds of action (reaching, grabbing, passing through doorways, sitting on chairs, etc.), and the typical errors seen in judgments of own ability follow consistent patterns. Understanding how perceived action boundaries differ from actual physical limits informs an essential question: how do we know what we can do? The current work investigates regular discrepancies between perceptions of towards things that appear at or near the limit of one's arm's reach, and actions taken towards the same items. These studies establish how and when these errors occur and whether they can be corrected through experience. Perception depends on the body's relationship to the environment, and to fully understand visual perception and how it guides action it is essential to understand both how individuals perceive the environment, and how they believe their body can interact with it.

\subsection{What are Action Boundaries, and why do they matter?}

James Gibson established the concept of affordances several decades ago: features of the environment afford certain kinds of action or use towards achieving certain goals, based on both the physical features of the environment and the physical features of the perceiver. The limits of the perceiver's body, then, inform what kinds of actions the environment affords him/her. By this 
account, a chair is not simply a 'chair,' but rather is perceived in terms of its own physical features (size, rigidity, height), and those of the perceiver. A chair may afford standing-on for a fully grown and healthy adult, but would not for a toddler just learning to walk, or an aged, arthritic, or injured person (Gibson, pp. 127). Simply put: when it comes to perception, actions matter.

It follows, then, that perceptions must be scaled not by arbitrary units (inches, pounds, miles, etc.), but to physical characteristics of the individual perceiver. No two individuals perceive the environment in exactly the same way, because no two individuals are, physically, identical. Action informs perception, and perception guides action.

Classic research identifies two types of points that define the relationship between one's body and one's environment: 'optimal points,' or the point at which the action in question is easiest or is perceived to be easiest, and 'critical points,' or the point at which one must switch from one type of action to another (Warren, 1984). Typically studies of action boundaries refer to the latter, because they mark the actual physical limit of the ability to act. Studies of critical points are much more common across the literature, examining whether or not people can accurately perceive their own action boundaries. For example, stairs are judged to be 'climbable' when the risers do not exceed a certain proportion of the individual perceiver's leg length (Warren, 1984), objects are perceived to be 'graspable' when they do not exceed a size that can be grasped by the observer's hand (Linkenauger, Witt \& Proffitt, 2011), and targets appear to be 'reachable' when they are within either the extent of the participant's arm, or the extent of the participant's arm while holding a tool (e.g. Carello, Grosofsky, Reichel, Solomon \& Turvey, 1989; Witt, Proffitt \& Epstein, 2005; Davoli, Brockmole \& Witt, 2011). These critical points 
mark the boundary of one's ability to successfully act in a given mode; they mark an individual's action boundaries.

\subsection{Action boundaries (and many other things) scale individuals' perception of the environment}

The methods listed above have been used to show that individuals perceive their environment based on their ability to interact with it - individuals perceive the opportunities that the environment affords for action. These abilities (and how they are perceived) depend not only on the individual's physical dimensions or morphology (Warren, 1984; Warren \& Whang, 1987), but in many cases on their skill and feedback about their performance (Witt, Linkenauger, Bakdash \& Proffitt, 2008; Lee, Lee, Carello \& Turvey, 2012), their emotional or psychological states (Graydon, Linkenauger, Teachman \& Proffitt, 2014; Canal-Bruland, Pijpers, \& Oudejans, 2010; Bootsma, Bakker, van Snippenberg \& Tdlohreg, 1992; Linkenauger, Lerner, Ramenzoni \& Proffitt, 2012), their motivations or goals (Witt, Proffitt \& Epstein, 2005), or their experience with a given action (Cole, Balcetis \& Dunning, 2013; Franchak, van der Zalm \& Adolph, 2010), and can change as physical characteristics (Wit, Proffitt \& Epstein 2005; Franchak \& Adolph 2014) and goals (Witt et. al., 2005) change.

Skilled performance augments perception of the size and speed of targets. Witt and colleagues found that golfers who putted more accurately would perceive the golf hole as larger than those who putted less accurately (Witt et. al., 2008). Similarly, football players who kicked field goals would judge the uprights to be farther apart and closer to the ground when they completed a series of more successful field goal kicks, but only if they made their size judgments after they kicked the ball (Witt \& Dorsch, 2009). Tennis players will judge that a ball was moving faster prior to hitting that ball out-of-bounds, than they would judge a ball moving at the 
same speed if they hit it in-bounds (Witt \& Sugovic, 2010). Skilled, competitive archers will judge that targets appear bigger when (after) they have shot more accurately (Lee, Lee, Carello \& Turvey, 2012). Unlike Witt and colleagues in their several studies, Lee and colleagues did not give their archers any feedback about their performance. Archers judged targets to be larger when they shot better without knowing how well they were shooting, suggesting that performance's effects on perception are not necessarily dependent on visual (or indeed, any) feedback.

Perceived affordances and action boundaries also change with experience. Individuals judge their capacity for familiar and more controlled actions better than novel or more dynamic actions, and both children and adults will more accurately judge the affordances of an environment after some practice. Adults made more accurate judgments of doorways' passability when they, first, practiced walking through different sized doorways several times (Franchak et. al. 2010). Throughout development, children with more experience moving in a specific way will make fewer errors when acting. When close to a gap between two tables with a significant dropoff, experienced walkers will correctly judge when the gap is too large to safely cross, while novice walkers, when walking, will attempt to step over an impossible gap (Kretch \& Adolph, 2013). Adults and older toddlers will also learn, after a single fall, the limits of their ability to pass over a narrow ledge (Joh \& Adolph, 2006).

While experience goes a long way toward making judgments of affordance more accurate, it does not capture the whole phenomenon. Cole and colleagues tested perceived affordances of a wider range of actions for crossing a gap: one common (leaping, stepping), some uncommon (swinging on monkey bars), and some common but disused (crawling). They found that participants more accurately judged the size of the largest passable gap when 1) the 
action they were to use was familiar and 2) when that action was also static, or more controlled. Participants were less accurate in their judgments when an action had dynamic qualities, like jumping or jumping and swinging from a bar. It is interesting to note that while jumping and swinging from a bar has the added uncertainty of being an unfamiliar action to most people, launching off of one leg to leap across a gap is quite a bit more familiar. Regardless of this familiarity, the study found that these action with dynamic qualities elicited greater estimation bias. Their findings suggest that while experience increases accuracy in judging affordances, the dynamic qualities of an action can introduce uncertainty that is difficult for participants to account for (Cole et. al., 2013). Presumably, the boundaries of dynamic actions are more difficult to accurately estimate because they are more difficult to know; they are inconsistent - the life's worth of feedback that you have is less helpful than it is for a more static parameter of your body.

Anxiety, a psychological state with a distinct physiological signature, also influences perceived action boundaries and perceived affordances. Inducing anxiety, in one study, led participants to estimate that they could not reach as far, relative to their actual arm's reach, as non-anxious participants. Participants who breathed through a narrow straw to reduce oxygen intake and create feelings of anxiety, estimated their arm's reach to be much shorter than participants who did not. Interestingly, non-anxious patients over-estimated their reach significantly, while anxious participants' estimates were much closer to their actual arm's reach (Graydon et. al, 2012). Canal-Bruland and colleagues argue that some high-anxiety situations can actually dampen participants' ability to perceive the environment in terms of its affordances. Participants threw darts at a target under circumstances that evoked different levels of anxiety (standing on a low or a high platform), and then judged the size of the target. Replicating 
previous work on action-specific perception, participants perceived and reported a larger target, but only in low and moderate anxiety situation. The judgments of participants in high-anxiety condition were not influenced by performance (Canal-Bruland et al, 2010). The authors suggest that anxiety increases the load on the attentional system to the point where participants cannot incorporate their performance into their perceived affordances. Anxiety increases cognitive load, leaves no capacity with which to incorporate abilities into one's action boundaries, and eliminates the effect seen in the low-anxiety condition. Perception of both one's action boundaries and the affordances of the environment are malleable, and can be influenced by psychological factors.

Autistic individuals do not accurately judge their environments' affordances, and make errors judging whether a given aperture is big enough for their hand to pass through, judging the reachability of targets, and judging the graspability of objects (Linkenauger, et al, 2012). This evidence suggests that accurately perceiving the relationship between one's body and one's environment is a function of typical development, which either does not occur or is delayed in autistic individuals.

Perceived action boundaries are flexible, and participants can 1) incorporate a tool into their own action boundaries in real time and 2) this flexibility impacts how distances are perceived in near space. Targets will appear to be closer when participants have a tool that extends their reach (Witt, Proffitt \& Epstein, 2004; Brockmole, Davoli, Abrams \& Witt, 2013), and this effect persists when participants estimate before they have reached with the tool (Witt et. al. 2004) if the participant simply imagines reaching with the tool (Witt \& Proffitt 2008; Davoli, et al, 2011), and when one watches someone else reach with a tool (Bloesch, Davoli, Roth, Brockmole \& Abrams, 2012). This flexibility of action boundaries has been observed with more 
gradual changes as well: pregnant women can judge the minimum passable width of a doorway with relative accuracy throughout pregnancy, and non-pregnant adults fitted with a prosthetic 'pregnant belly' (Franchak \& Adoph, 2014). The over-arching point illustrated by this work is that knowledge and perception of affordances can change in real-time. Individuals are sensitive to changes in their physical capacities, even without practice; this in turn changes what they think they can do, and how they see the world.

Perceived affordances are malleable and reliant on both the actor and the environment. In several illuminating cases, they are also inaccurate. These errors provide insight into the mechanisms that drive the perception of action boundaries and affordances.

\subsection{Methodology - how do we study action boundaries?}

Action boundaries have been studied using relatively simple methods that all aim to determine what people THINK they can do, and then compare that to what they can ACTUALLY do. These methods typically fall into one of two broad categories: 1) viewing a series of stimuli of different sizes/at different distances and judging whether they afford a specified action, or 2) adjusting a token to match the perceived limit of the action in question.

In the first kind of study, participants are shown a series of targets or items, and asked whether or not they afford a particular action for the participant. Warren (1984) examined the perceived climbability of stairs using this type of method: Participants repeatedly viewed a set of five stair heights and responded gave a yes/no response to whether they could step up the stair "in the normal way, without using your hands or knees." Franchak and colleagues used a similar forced choice paradigm to examine the perceived passability of doorways by showing participants a series different-sized doorways one by one and asking whether they thought they could pass through the doorway by "turning to the side and squeezing into the doorway." 
(Franchak, van der Zalm \& Adolph, 2010). Franchak and colleagues enhanced the method by adjusting the stimuli so that they would, by the end of the study, center on the participant's affordance threshold. Using a similar method, Heft (1993) showed participants a series of targets and, again, asked for a yes/no response about the target's perceived reachability. Rather than adjust target distances throughout the study, Heft measured actual reach prior to the rest of the study, and centered the target distances for each participant on their actual action threshold.

In a second type of method, the experimenter will present the participant with a slowly moving or changing target and ask the participant to say 'stop' when the target reaches the limits of what they believe they could successfully act upon. When measuring perceived reachability over a table, Graydon and colleagues (2012) slid a small disc across a table that the participant was seated at, along five different trajectories $\left(30^{\circ}\right.$ contralateral, $15^{\circ}$ contralateral, straight ahead, $15^{\circ}$ ipsilateral, and $30^{\circ}$ ipsilateral) and participants would report their estimates by telling the experimenter to stop when they reached their perceived limit.

Other methods assess perceived action boundaries indirectly by observing and quantifying actual behavior. For example, Kretch and Adolph (2013) placed children at the edge of a gap in a table, and presented them with objects on the other side of the expanse. The experimenters manipulated the width of the gap, and observed under what conditions children would attempt to reach for the object from both familiar and unfamiliar postures - seated, crawling and standing (Kretch \& Adolph, 2013). Using these kinds of open-ended methods, researchers can learn about not just what people think they can do (perceived action boundaries) and what they can actually do (actual action boundaries), but what they DO do. In an even less constrained example, Gonzalez and colleagues sat participants at a table strewn with legos, provided them with pictures of lego models, and asked the participants to build what they saw. 
By observing unconstrained reaching and grasping behavior, they discovered that left handed individuals do not favor their left hand when making repeated precision grips (Gonzalez, Whitwell, Morrissey, Ganel \& Goodale, 2007). These methods offer insights into how physical limits (and lack thereof) influence actual actions in a more ecologically valid way, and they implicitly acknowledge that what a person CAN do and what they ACTUALLY do are not always the same.

The most extensive work in this area has concerned perceived reachability, presumably because reaching is relatively simple to manipulate and measure. Still, methods in such studies encounter subtle issues that have, over time, been themselves manipulated to inform and characterize the process of perceiving affordances. The Degrees of Freedom problem (Bernstein, 1967) notes that action complexity increases as the number of mobile joints involved in the action of interest. Reaching, a seemingly simple action, necessarily depends on the range of motion of the joints in the finger, the wrist, the elbow, the shoulder, and the participant's ability to bend at the waist. To control the experimental set-up, researchers have typically restricted participants' range of motion, most typically by requiring that participants maintain upright posture and do not bend at the waist. Over time, researchers have also meticulously manipulated the kind of physical position that participants assume while judging reachability, in an effort to understand the role of postural stability in affordance perception. In many studies, reachability judgments are made from a seated position while targets appear on a table in front of the participant (e.g. Carello et. al. 1989; Heft 1993; Witt, Proffitt \& Epstein 2005). Others have manipulated postural stability and physical degrees of freedom by asking participants to judge reachability while standing, balancing on one leg, or laying supine, while targets appeared in 
front of, directly to the side of, of above the participant's head (Carello, 1989; Rochat \& Wraga 1997; Fischer, 2000, Gabbard et. al. 2005).

In addition to the participant's position, researchers have tinkered with how they prompt participants to hold said position. Instructions must explicitly define what experimenters mean when they instruct participants to 'reach,' in order to achieve consistency across participants. As mentioned above, some studies allowed participants to 'bend naturally at the waist' when actually reaching, and encouraged them to think of the action in this way when estimating their ability (Heft, 1993), while others request that participants sit up straight and keep their back in contact with the back of the chair (e.g. Carello et. al, 1989; Rochat \& Wraga, 1997; Bootsma et. al., 1992), while still others physically restrain their participants by doing things like clipping their shirts to the chair they are seated on (Witt, 2008), or strapping them in using a modified car safety belt (Gabbard \& Ammar, 2005). In one study conducted on participants while standing, Rochat \& Wraga (1997) secured participants' feet to the surface on which they stood. These methods were all undertaken with a goal of reducing participant uncertainty about the task, and generating cleaner data. It is possible, though, that restraining participants could, itself, influence their perceived abilities.

Across the several kinds of action and affordance studied, these studies all examine accuracy - how close are the participants' perceived action boundaries to their actual action boundaries. These studies have been conducted based on the assumption that participants can accurately and consistently judge their own action boundaries. This has been confirmed through the years; participants, when asked, will report their perceived action boundaries with high levels of confidence and little to no hesitation. This research has, though, elucidated consistent patterns of error. 


\subsection{Perceived and Actual action boundaries are not always the same - and there are several accounts suggesting why}

Studies have repeatedly shown that participants' estimated action boundaries are inaccurate in consistent ways, which appear to be heavily dependent on the features of the experiment, or the context of the judgment being made. Distinct patterns of response bias emerge, depending on how and where participants are asked to judge the limits of their affordances.

On occasion, studies of reaching find consistent under-estimates. Gabbard and Ammar observed that participants underestimated their arms' reach when using binocular visual cues, while participants very slightly over-estimated reach with monocular cues (Gabbard \& Ammar, 2005). Some experimental circumstances have elicited more accurate affordance estimates from participants. When participants judged the reachability of targets on a high table - the table appeared at about chest-height for all participants - their judgments were much more accurate than when the table was at waist height or lower (to which they over-estimated) (Carello et. al., 1989). Heft found that reachability estimates were more accurate when speeded, or when participants did a cognitive task in between viewing the target and reporting their judgment (Heft, 1993).

Most commonly, though, experimenters have observed over-estimation of action boundaries; participants believe they can act beyond their physical limits. Heft observed significant over-estimation when his participants had unlimited time to make their responses (1993), and Gabbard and Ammar saw participants over-estimate when judging the reachability of objects presented at their midline (2005). Carello and colleagues observed participants overestimate their arm's reach when targets were shown on a table that was both low (below waist 
height for standing participants) and at a normal height (1989). Bootsma and colleagues found that participants consistently over-estimated their ability to reach targets as they moved quickly in front of them (1992). Participants over-estimated reach when presented with overhead targets while standing upright, and while shown targets in front of and to the side of themselves when standing on both one and two feet (Fischer, 2000). Finally, Graydon and colleagues (2013) saw all participants over-estimate their arm's reach when targets were presented at the midline or at varying angles on the side of the table contra-lateral to the arm to-be-reached with, regardless of anxiety levels (although anxiety did reduce the over-estimation bias). This over-estimation bias appears through a wide range of studies and seems relatively persistent.

These studies all beg the question: where does over-estimation bias come from? The three accounts below illustrate the conclusions of the body of research done on the topic. Harry Heft argues that cognition, not perception, is to blame for errors in perceived reachability (1993), while Rosenbaum and others suggest that errors arise from the stability of the perceiver's physical position at the time the judgment is made (Carello et. al, 1989; Gabbard, Cordova \& Lee, 2007), and still others argue that inaccuracy occurs because it is impossible for individuals to estimate affordances without taking their whole body's movement potential into account (Rochat \& Wraga, 1997; Robinovich, 1998).

\subsubsection{Post-Perceptual Interference}

Harry Heft's work proposed that over-estimation bias is cognitive, not perceptual. Perception, he argued, would accurately reflect a body's abilities, but that allowing participants unlimited time to judge whether or not a target was reachable was compromising the accuracy of participant judgments. Unlimited time allowed cognitive influences, adjustments, and compensation to warp the reporting of otherwise sound perceptual information. He suggested 
that people are much more accurate when they report without thinking. Inaccuracy, by this account, is an artifact of post-perceptual processes.

This conclusion follows a study (discussed briefly in sections above) in which participants are asked to respond, yes/no, to whether a series of targets is within reach in one of three conditions. In the first, participants are forced to respond within a fraction of a second after the presentation of the target. In the second, participants are allowed as much time as they would like to respond. In the third, participants are given time to make their responses, but they are required to do a cognitively taxing task in between the moment that they see the target and the moment that they respond. Responses were most accurate in conditions one and three, and an overestimation bias was evident in condition two (Heft 1993).

While Heft presents a solid account, other researchers have elicited reduced overestimation bias using other means. For example, Graydon and colleagues found a significantly reduced overestimation bias when they were asked to judge the limits of their arm's reach on the ipsilateral side of the table - they over-estimated their right arms' reach less when they were asked how far they could reach to the right side of a table (Graydon et. al., 2013). Additionally, over-estimation is reduced when participants judge the reachability of objects while lying supine, and presented with targets off to one side of their body, even with their shoulder (Fischer, 2000). Both of these studies allow participants unlimited time to judge reachability, suggesting that cognition is not always the enemy of accurately reported perception. Finally, participants can accurately judge affordances related to other action boundaries given ample time, too. While participants over-estimate the width needed to successfully walk through a doorway, they can accurately judge the minimum passable height of doorways, given unconstrained reporting time 
(Cole et. al., 2013). It is unclear why perception of some affordances would be selectively distorted by post-perceptual processes, while others would not.

\subsubsection{The Postural Stability Hypothesis}

This Postural Stability Hypothesis suggests that individuals are more conservative when estimating abilities from an unstable posture (standing on one leg versus lying down, fully supported by the floor). Gabbard, Cordova \& Lee (2007) argue that perceived reaching limits depends on an individual's perceived postural constraints. They asked participants to report perceived reachability of targets while standing on one leg and while seated, and compared estimation errors between the two conditions. In both conditions, after the experimenters measured actual reach, they showed participants a series of targets, whose distances were centered on participants' actual reach. They found a significant over-estimation bias in the seated condition, while (not-significantly) under-estimation bias in the standing condition (Gabbard et. al., 2007). They conclude that a greater demand on one's own system of postural control leads to more conservative estimates of one's abilities, generating more conservative estimates, and they suggest that confidence is a driving mechanism behind this effect. Increased stability increases participants' confidence that they can accurately judge their abilities. On the flip side, decreased postural stability results in more uncertainty about one's abilities and, in turn, more conservative estimates.

In addition, Robinovich (1998) finds that while participants do consistently over-estimate their reach while standing upright against a wall, they will likewise underestimate the extent of their reach while standing and leaning forward. Participants were instructed to estimate the farthest extent of their arms' reach either while standing with their heels and back flush against a wall, or while leaning forward and keeping their heels firmly on the ground. Robinovich 
suggests, like others, that instable starting postures will cause participants to make more conservative estimates of their abilities due to the increased possibility of a fall, or of failure in completing the given action.

This relates to work that distinguishes between static and dynamic motions. Robinovich and colleagues use slightly different language, but they find (in several different types of action) that participants are more conservative (usually less accurate) when judging the limits of a dynamic or changeable, rather than a static or more controlled, action. As mentioned above, participants are consistently conservative when judging how wide a doorway must be in order to successfully walk through it, but they will be accurate when judging how tall the same doorway must be. The authors argue that this occurs because walking is a dynamic action, during which the actor can sway side to side, squaring and un-squaring shoulders with the target destination, while height remains relatively constant whether the participant is moving or not (Franchak, Celano \& Adolph, 2012). Posture is less stable and more prone to change during dynamic acts, and participants allow for some 'wiggle room' when estimating the limits of these abilities (or the limits of different features of their bodies when completing these abilities).

Some developmental work also supports this claim, showing that children who were more experienced at sitting upright independently would more accurately judge whether or not an object was within reach. As children develop the ability to sit independently, they also develop enhanced coordination, stability, and control of their torso and upper arm movements while in a seated position. Infants who were eithe non-sitters, inexperienced sitters, or experienced independent sitters were presented with objects that were within reach, just at the limits of reach, and beyond reach. Results showed that experienced sitters were the only ones who would consistently execute successful reaches (as opposed to failed reach attempts) towards 
more distant, but still reachable, objects. The researchers suggest that infants will both be more successful at completing actions and at judging when actions are complete-able (not attempting impossible actions) when they are more able to control and stabilize their bodies while reaching (Rochat, Goubet \& Senders, 1999).

\subsubsection{The Whole Body Engagement Hypothesis}

A final account for inaccuracies in estimated reachability has been named the 'whole body engagement hypothesis,' and its name is on point in describing its content. The accounts primary proponents suggest that the body has a number of different dimensions by which it extents, contracts, and moves when making everyday movements. People estimate their abilities, this account suggests, while accounting for all of their physical degrees of freedom. Estimates are inaccurate because per usual experimental methods, participants are asked to estimate reaching ability from a semi-restricted posture. They are unable to suppress their knowledge of their full range of motion when imagining themselves completing the action, and so overestimate when asked (Rochat \& Wraga, 1997).

In addition, Robinovich, who is mentioned above suggesting a postural stability account, goes on in his 1998 paper to suggest a whole-body explanation for why individuals over-estimate their reach while standing upright. He points out that reaching in everyday life usually involves some degree of forward lean, an action that is expressly prohibited during this study. It would be only natural for participants to revert to this more natural posture when estimating an ability called 'reaching.' The data from his study suggest that factors other than individual physical dimensions (height and arm length) have significant influence over estimated reach, but he stops short of identifying what those parameters might be. 
The latter two hypotheses - postural stability and the whole body engagement hypothesis - were put to the test in two studies by Fischer in 2000, and ultimately concluded that neither account reliably predicted behavior. In study 1 , Fischer compared reachability estimates for targets that were either displayed above the participant's head or to their side, either while they stood or lay supine. The postural stability hypothesis would have predicted that participants would over-estimate the most when judging abilities used from a stable position (lying down) when compared to a standing or otherwise less-stable position, while the whole body engagement hypothesis would predict that postures with greater freedom of movement and farther possible physical extension would result in the greater over-estimation. Contrary to the postural stability hypothesis, Fischer found that participant over-estimated the least when they estimated their abilities while lying supine. In study 2, participants judged reachability of targets presented either to their right or their left whiles standing on their right, left, or both legs. Postural stability would suggest the greatest over-estimation in both of the one-legged conditions, while the whole-body account would predict greater over-estimates when the target appears on the same side as the foot the participant stands on: the participant could use the free leg as a counter-balance, and lean much farther than they could in the other direction. Results did not fully support either account: there was no main effect of stance OR position of the target. In fact, participants slightly (non-significantly) under-estimated their reach in all conditions. Taken together, these results suggest that while both postural stability and the tendency of participants to simulate actions with their whole body can influence perceived affordances, neither is solely responsible for reports of perceived reach (Fischer, 2000).

All of the above accounts suggest elegant, conceptually complex explanations of this error, but none definitively provide a solution. These studies have several methodological 
elements in common, however, and one or more of these elements could be driving this effect. Before we can argue for a conceptual account of what is happening, it is necessary to rule out the simple, methodological issues that could play a part.

\subsection{How do we know our action boundaries?}

Whether or not estimates are accurate, individuals can readily estimate the limits of their abilities. This bodily knowledge, and this sense of how one's body fits within and interacts with features of the world, is learned by both children and adults through active exploration. Work from both developmental and adult perception/action literature focuses on the visual and proprioceptive information gleaned by moving, successfully and otherwise, throughout the world, and highlights the importance of practice and action-specific experience in developing an accurate sense of how the body and the world interact. Under most circumstances, people can maintain an (relatively) accurate sense of their abilities in an environment, and can keep this sense of their body updated with relevant and salient experience.

Classic works like Held and Hein’s (1963) ‘kitten carousel’ experiment, and work with infants and the visual cliff, have made it known for decades that accurate visual perception of the world depends on an infant's/toddler's ability to locomote through it (Adolph, Bertenthal, Boker, Goldfield, \& Gibson, 1997). Active exploration also shapes visual behavior in children. Young children who could sit independently, but who could not yet reliably grasp objects, were either given 'sticky mittens' that allowed them to lift and move objects around them, or watched as a caretaker manipulated those same objects. After two weeks of training, the children who used 'sticky mittens' displayed visual tracking and following behavior much like older children who could grasp objects on their own (Libertus \& Needham, 2010). Physical experience influences visual behavior, and ostensibly changes the kind of visual information the child gets about the 
world around him/her. Experience also shapes how infants choose to (or, in some cases, not to) navigate the world, and through development children become better at using information from failed attempts at action. Children develop the ability to learn using integrated visual and proprioceptive feedback.

Infants learn of the world, and their body's place in it, through active exploration, and studies have shown that this learning extends only to the modes by which they can independently explore the world. In three studies, some children would attempt to walk down slopes that would have afforded walking with high friction underfoot, but would fall in a lower-friction condition repeatedly, showing very little learning. Infants could, though, glean information about the slope's affordances through direct tactile exploration in concert with visual cues: infants were less likely to traverse a too-steep slope if they had visual information about its steepness (studies 1 and 2), or if a change in the appearance (color) of the surface indicated a change in its friction (study 3) (Adolph, Joh \& Eppler, 2010). Children appear to understand the laws of physics that govern the world around them - that if they went over a cliff they would fall and hurt themselves - but this understanding is limited to locomotor methods by which the infant can independently move. For examples, non-walkers (or inexperienced walkers) who can crawl proficiently will NOT attempt to crawl across a gap that is too large to pass, but if they are placed upright on their feet and prompted to walk, they will attempt to walk (and fall) through the same sized gap (Kretch \& Adolph, 2013). Once children are experienced walkers, they will no longer attempt to walk across impossible gaps, although they will try different strategies to climb safely down in order to reach the other side. Experience definitely enhances a child's understanding of their body's place in the world, but they are learning locomotion-specific relationships, rather than general physical laws about the world. 
Joh and Adolph also found that the ability to learn develops with age; children can learn from experience, but they learn much more slowly than adults. They tested the adaptability of children of different ages as well as adults to walking over a surface with changeable stability. The walkway used in the experiment contained a deform-able section made of soft foam, that was visibly distinct (by color and pattern) from the solid portion of the walkway. Experimenters observed how many trials of walking over the walkway it took before participants could do it without falling. Young children who were newer walkers were hesitant to traverse an unfamiliarlooking surface, and would take much longer to learn how to modify their movement to avoid falling. Once children reached 39 months, however, they learned and avoided falling after a single trial, much like their adult counterparts (Joh \& Adolph, 2006).

Adults also learn through active exploration - actual action boundaries change depending on the environment and one's physical state. As discussed above, practice walking through doorways of different size enhances accuracy in judging the smallest possible doorway that one could successfully pass through (Franchak et. al., 2010). Task-specific experience attenuated judgments of participants' relationship to the environment. Additionally, Joh and colleagues investigated the degree to which proprioceptive information interacts with visual information to inform judgments of environmental features (Joh, Adolph, Narayanan \& Dietz, 2007). Specifically, they investigated how different levels of friction underfoot, and direct experience with that friction on a slope, would bias estimates of a hill's steepness. They determined that decreased friction (and a greater likelihood of falling) increased estimated slope, and increased friction induced the opposite effect. Most interesting, in the context of the current discussion, is the effect of experience. In all of the five studies, participants stood on the surface of greater or lesser friction while looking at and judging the slope. Slope estimates only became more 
accurate when this experience of the friction underfoot happened in concert with immediate, physical contact with the slope to be judged. Here, active exploration increased accuracy in judgments of the environment, but only when that experience was directly and specifically related to the feature being perceived and appraised (Joh et. al., 2007).

In keeping with these findings, it has been shown that pregnant women can accurately judge the passability of doorways, even as their bellies continue to grow. Across a pregnancy, previously passable doors become impossible to squeeze through, but women will not attempt to fit through doorways that will not accommodate their growing bellies (Franchak \& Adolph, 2014). These researchers also found that non-pregnant adults can accommodate new girth into their perceived action boundaries, to almost the same degree of accuracy as their pregnant counterparts. Non-pregnant adults wore a pregnancy simulating prosthesis, and judged whether or not they could fit through a series of doorways either without (Study 2) or with (Study 3) practice. In Study 2, participants judged the passability of, and then choose whether to attempt to walk through, doorways of several sizes. While non-pregnant participants wearing the prosthesis were much less accurate (and conservative, declining to pass through doorways that they could have physically fit through) at the start of the trials, their accuracy increased with practice. In the third study, they confirmed that participants poorly integrate the prosthesis into their body schema without some kind of practice or feedback. Once participants have even minimal feedback about how their new bodily dimensions interact with the environment, estimates of abilities improve dramatically (Franchak \& Adolph, 2014)

Practice has different effects on performance and judgment, though, depending on the kind of motion being undertaken. As mentioned above, adults and children (especially after a certain age) will learn from a single fall a) the limits of their body in that environment and b) that 
alternate locomotor strategies should be tried to overcome those limits (Joh \& Adolph, 2006). Participants also develop more accuracy in judging the minimum necessary width of a doorway with practice (Franchak, van der Zalm \& Adolph, 2010). Individuals can also learn the minimum necessary height of a doorway when moving by a different mode - in a wheelchair - but recalibrating one's judgments to this new method of locomotion takes several days of practice (Higuchi, Takada, Matsuura, \& Imanaka, 2004). Individuals do not, however, re-learn their body's relationship to the environment when they change their height by wearing platform shoes. Participants could not learn the new limits of their sit-ability, and consistently under-estimated the maximum possible height of a chair that they could successfully sit on, even after several rounds of practice (Mark, Balliett, Craver, Douglas \& Fox, 1990). Finally, individuals can accurately judge the size an opening must be for their hand to fit through, regardless of whether it is their dominant or non-dominant hand that will do the reaching (Ishak, Adolph \& Lin, 2008). When individuals are already relatively accurate, practice does not enhance their estimates.

In some cases, practice does not seem necessary for accurate recalibration. Action boundaries change as the dimensions of one's body change (both naturally and artificially), and studies have shown that knowledge of one's action boundaries can change without practice. As the tool-use effect shows (Witt, Proffitt \& Epstein 2005), individuals can incorporate new elements into their action boundaries, and as a result their perception of their surroundings will change. Importantly, while this tool-use effect does not require direct experience using the tool, it does require intent. The same study demonstrated that the effect does not occur when the participant does not either imagine reaching or plan to reach with the tool (Witt, Proffitt \& Epstein 2005). 
Both children and adults can adapt their actions and perceived capacity for action to changes in their body's dimensions and abilities. Participants are accurate when asked to decide whether they could (and whether they will attempt to) fit their hand through an aperture, choosing not to act when the opening is too small to fit through. Interestingly, here, practice (or, more accurately, experience) does not enhance the correspondence between action and affordance - participants' actions were in accordance with their action boundaries equally with both their dominant and non-dominant hands. Put another way, their accuracy could not increase from non-dominant to dominant hand because their performance was already at or near ceiling. Additionally, when these participants wore a prosthesis that enlarged their hand, their decisions remained accurate to their new abilities. These participants were successfully able to adjust their known action boundaries, even without practice (Ishak, Adolph \& Lin, 2008). While this seems at odds with the other work discussed in this section, remember that the action tested in this study was simple, common, and static. Other studies show the greatest benefits of practice improvement in the accuracy of judgments - when actions are dynamic and outcomes are less consistent and certain. Participants were already accurate at this simple task, so performance would not likely improve much with practice, and reaching through an aperture is an action that can be controlled and adjusted throughout execution. This finding begins to identify some limits of improvement that follows practice.

The dynamic qualities of an action can influence accuracy of one's judgments: it is more difficult to accurately predict the outcome of dynamic actions, even when they are familiar and common. As mentioned in the above section, participants' past familiarity with an action does not result in more accurate judgments of one's action boundaries if that ability is dynamic, like launching off of one foot to traverse a small gap (Cole et. al., 2013). This again illustrates a limit 
of the benefits of life experience (not necessarily practice) on perceptions of action boundaries. Actions with more dynamic components have more variable outcomes, and are in turn more difficult to estimate accurately and with low variability.

As the literature above displays, knowledge of physical abilities and action boundaries result from active exploration, practice, and life experience. Children learn these relationships between their bodies and the environment in a modality specific way (a way specific to a particular kind of locomotion like walking), and become better at learning from experience as they age. Both adults and children, under most circumstances, use practice to fine-tune their known action boundaries, and to update their understanding of their bodies' dimensions. For the most part, this work converges nicely on the attenuating properties of practice, and the possibly mediating effects of physical stability/dynamics of action.

Rochat and Wraga note that "errors in the perceptual judgments of what is reachable are certainly not attributable to a lack of learning opportunity" (1997), which elegantly sums up the research presented in this section. Both children and adults constantly and dynamically interact with the environment, and this informs both perception and future actions. While the bulk of the research presented above suggests that accurate body knowledge comes from action-specific experiences over time, a few qualifiers to this effect become evident as well: young children do not learn to the same degree (or at the same speed) as older children and adults, the consequences of dynamic actions are learned and understood differently than those of static acts, and information about the limits of an action (or a feature of the environment) gleaned through exploration does not generalize to other kinds of action. Experience informs body knowledge, but only within limits. 
One common feature of this research is the assumed role of visual feedback in the learning process. Researchers take it as known that affordances will only become known, in children and adults, if faithful visual feedback accompanies this experience. While studies like the kitten carousel do strongly link healthy visual development to active exploration, the same link has not been made between visual experience of one's body, and one's knowledge of that body's abilities. Future research is needed to examine the degree to which developed knowledge of action boundaries depends on vision.

\subsection{What is known and what is left?}

People perceive the world in terms of their body's ability to act, even while those bodies, their environments, and their owners' intentions change. While people will confidently report the limits of their own abilities, those estimates are often inaccurate, with individuals tending to over-estimate their body's abilities. The degree of these inaccuracies changes depending on the study, but over-estimation of reach is one of the most consistently observed phenomena in the reaching literature. The literature does not converge on a single explanation - in fact, it favors a multi-faceted approach - and questions still remain. Some methods typical to reaching experiments have yet to be fully examined with regards to their impact on over-estimation bias, such as the connotations of the instructions given to participants, and the method by which they are encouraged to maintain their upright posture. Before accepting a more conceptually nuanced account of the source of over-estimation bias, it is vital to determine whether it is simply an experimental artifact.

Practice and life experience tend to result in more a more accurate sense of one's abilities, although a few studies highlight exceptions to this trend. Work to date does not, however, address the role that vision plays in the learning and knowing one's physical limits. 
The present work begins to untangle the roles that proprioceptive and visual feedback play in this learning, respectively, to determine if visual feedback is necessary for one to more accurately (and with less variability) judge, for example, the reach of one's arm. 


\section{Study 1: Are Decisions about Affordances Different at Action Boundaries than Within or Beyond these Boundaries?}

\section{Introduction}

To begin an investigation of decision-making at and around action boundaries, it is necessary to establish if decisions made about actions at and around the action boundary are somehow different than decisions made about one's ability to act on targets either comfortably within, or markedly beyond such a boundary.

We accumulate a lifetime's worth of experience reaching out our arms and manipulating the objects around us. This experience lends us to a general understanding of our physical limits; when objects are very close we can easily identify that they are within reach, just as we know that objects across the room are definitely beyond reach. The space at and around our limits presents a more difficult task, however, because when objects appear at and around the boundary of reach, broad knowledge gleaned from experience may be less certain and judgements may be relatively more difficult to make.

The present experiment examined the pattern of response times that occurs when people judge the reachability of targets that appear at a range of distances. Participants were shown a randomly ordered set of targets on a table-top at distances ranging from very near to clearly out of reach. On each trial, participants decided whether or not they could reach the target with their right hand, and responded yes/no on a keyboard with their left hand. In one condition, participants would be asked to judge the reachability of each target with their right hand, and in the other, they would be shown a tool that extended reach (a conductor's baton) and asked to judge the reachability of targets while holding the tool in their right hand. Their reaction times were recorded, and we expected that reactions to targets at and immediately around the action boundary would be slower than those to targets both easily within reach and obviously beyond 
reach. This anticipated result would imply that decisions about the affordance of reaching are more uncertain at the action boundary for reaching.

\section{Purpose}

This study assessed whether reaction speeds slowed at and around individuals' action boundaries, as well as increased rates of error.

\section{Method}

\section{Participants}

73 University of Virginia undergraduates participated in this study for course credit. 10 were excluded for failing to follow directions, or for inability to complete the study due to equipment malfunction (stats), resulting in a total $\mathrm{N}$ of 63 (42 women, ages 18 - $23(\mathrm{M}=19.13$, $\mathrm{SD}=1.09) 59$ right-handed).

\section{Design}

The study comprised a one-way within participants design (Tool v. No Tool), examining participants' estimates of target reachability with and without a reach-extending tool, at varying distances both within and beyond the extent of the participant's reach. Reach was defined as the forward extent of the arm while the participants sit upright with shoulders parallel to the near edge of the table. Participants were informed, and reminded, of this definition throughout the experiment.

Participants sat at a table onto which targets were projected at varying distances, and were asked to judge whether they could reach the target with their right hand. Participants completed two counterbalanced blocks of fifteen randomized trials. Each block displayed the 
same set of fifteen target images; participants saw the same set of targets in a different order in each block.

Participants responded either 'yes' or 'no' via key press with the left hand, using the left and right arrow keys on a standard qwerty keyboard, respectively. In one condition, participants were asked to make this judgment about the reach of their right arm ('No Tool'), and in the other, participants were shown a tool that extended reach (a conductor's baton) and asked if they could reach the target "while holding the tool in their right hand" ('Tool'). At no point during the experiment were participants allowed to pick up or use the tool, nor were they given the opportunity to reach their arms out over the table. Response times were calculated as the time from the onset of the stimulus image to the participant's response.

After completing the estimated reachability trials, the experimenter measured both the actual extend of the participants' reach, and the participants' own estimate of their reach, both with the tool and without.

\section{Materials}

Each trial consisted of a single target (a white dot, approximately $2 \mathrm{~cm}$ in diameter on a black background). Participants were shown each of fifteen targets, each at a different distance, once per block of trials (twice total during the experiment). The closest target was shown 15.5 $\mathrm{cm}$ from the edge of the table nearest the participant, and the other targets got progressively farther in intervals of $6 \mathrm{~cm}$ (the next appeared at $22 \mathrm{~cm}$, the next at $28.5 \mathrm{~cm}$, and so forth).

The experimenter marked this point using E-Prime, which recorded the $\mathrm{X}$ and $\mathrm{Y}$ coordinates of a cursor, clicked at the farthest point to which each participant estimated that they could (or actually could) reach to. 
Experimental stimuli were programmed using E-Prime software (Psychology Software Tools, Inc. [E-Prime 2.0]., 2002). Stimuli were projected from an overhead-mounted projector down onto a white table top. The tool participants made their judgments regarding the extent of reach with a conductor's baton $45.7 \mathrm{~cm}$ long. Analyses were completed using R open-source statistical software.

\section{Procedure}

After providing informed consent, participants were instructed to sit at a table, and keep their hands in their lap for the duration of the study until explicitly asked to do otherwise. A series of slides with instructions were projected onto the table, and read aloud by the experimenter. The participant was told that the study was designed to test their spatial awareness, that they would be shown a series of dots on the table in front of them, and that, on each trial, they should judge whether or not they could reach each dot. Reach was defined, to the participants, as "the extent of your arm, while seated up straight with shoulders parallel to the edge of the table." Participants were reminded of this definition prior to each block of trials.

They were asked to respond, as quickly and accurately as possible, either 'yes' by pressing the right arrow key and 'no' by pressing the left arrow key on a keyboard placed next to their left hand. In the 'No Tool' block, participants were asked "could you reach the dot with your right hand?" and responded accordingly. At the beginning of the 'Tool' condition, participants were asked "could you reach the dot while holding the tool in your right hand?"

Following both experimental blocks, the experimenters collected data both about how far the participants thought they could reach, and how far they could actually reach. To record the former, the experimenter moved a dot away from the participant at a constant speed, and the participants were instructed to stop the experimenter when the dot reached the farthest point to 
which they thought they could reach. Estimated reach was measured twice, once for the "No Tool" condition, and once for the "Tool" condition. Finally, participants were asked to reach out and touch the farthest point on the table they could reach, first with their right hand and then with the tool, and this point was recorded using E-Prime.

\section{Results}

Reaction time data were inverse transformed into response speed (1000/RT) to correct for skew. Transformed data were analyzed using multiple paired-samples t-tests to compare reaction times of responses made to near targets, far targets, and targets that were shown at and around the action boundary. Data were collected in two conditions, one in which participants judged target reachability with their right hand, and one where they judged reachability with a tool. For the purposes of the current analysis, the data were collapsed across condition and considered together. 'Boundary' space was defined as the space from $85 \%$ of the participant's actual reach, to $125 \%$ actual reach. This space is centered just beyond measured actual reach due to the tendency of participants to over-estimate reported perceived reach. 'Near space' was everything within $85 \%$ of participant reach, and far space was bounded between $125 \%$ and $350 \%$ of participant reach. Responses beyond $350 \%$ of reach were sparse, as only a small number of participants had short enough arms for the far targets to appear that far away.

In keeping with the hypothesis, participant responses to targets shown within action boundary space $\left(\mathrm{M}_{\text {speed }}=1.16, \mathrm{SD}_{\text {speed }}=0.543\right)$ were slower than those made to targets shown in near space $\left(\mathrm{M}_{\text {speed }}=1.52, \mathrm{SD}_{\text {speed }}=0.53\right), \mathrm{t}(873)=11.57, \mathrm{p}<.001$ and in far space $\left(\mathrm{M}_{\text {speed }}=1.30\right.$ , $\left.\mathrm{SD}_{\text {speed }}=0.50\right), \mathrm{t}(899)=4.16, \mathrm{p}<.001($ See Figure 1.1). 


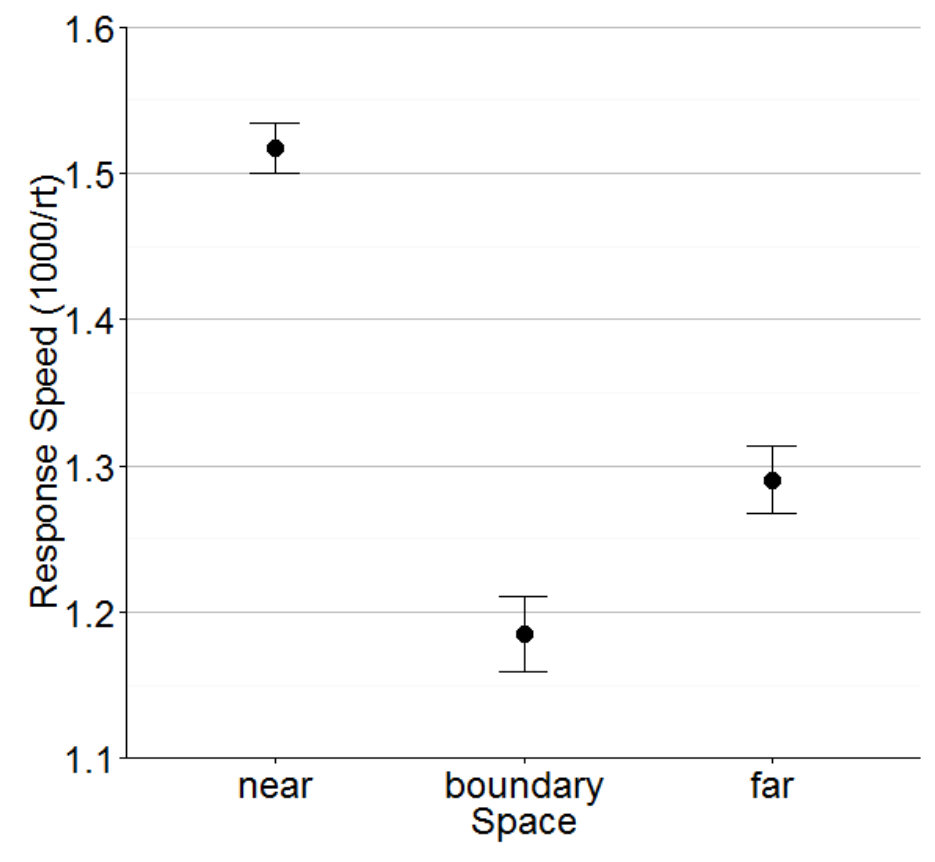

Figure 1.1. Response speed (1000/rt) as a function of Participant-relative space in which the target was presented.

\section{Discussion}

Participants responded more slowly to targets that were shown at and immediately around the action boundary than they did to either targets shown clearly within reach or clearly beyond reach. Participants also made significantly more errors when judging the reachability of targets at and around their action boundary. Taken together these results indicate that deciding how to act on targets shown near one's physical limits is different in some essential way. Possibly, these slowed reactions result from uncertainty; participants do make greater errors within the area around the action boundary. Additionally, it may be that slowed reaction times resulted from mental simulation; when participants were uncertain about their ability to perform an action, they utilized action-planning neural circuitry to simulate executing the action prior to making a decision (Grade, Pesenti \& Edwards, 2015). 


\section{Study Set 2: Over-Estimation Bias is Not an Artifact of Experimental Methods Introduction}

Decades of research using a variety of experimental methods show that participants overestimate their arms' reach. This over-estimation bias appears to varying degrees in the literature and researchers have manipulated the experimental set-up in attempts to discover why this bias occurs so regularly. Researchers have generated three accounts that offer explanations for the presence of over-estimation bias in some conditions and its absence in others, but ultimately, questions remain.

One account suggests that over-estimation results from top-down post-perceptual processes; perception is not biased, rather the observed over-estimation bias occurs when participants have too much time to think about their responses (Heft, 1993). Heft presents compelling evidence, but the fact that other studies have reduced over-estimation bias using nonspeeded methods by manipulating other features of the task (Cole et. al., 2013; Fischer, 2000; Graydon et. al., 2013) suggests his account does not fully explain the phenomenon.

The postural stability hypothesis proposes that individuals report more conservative estimates of their abilities when they make their judgments regarding an action that provides less physical stability. For example, participants should over-estimate less when judging their arm's reaching ability while standing on one foot, versus two (Gabbard, Cordova \& Lee, 2007). A second explanation - the whole body engagement hypothesis - suggests that individuals cannot estimate their body's potential to act without considering movement along every possible physical degree of freedom, even when explicitly instructed not to do so (Rochat \& Wraga, 1997). One researcher tested both of these accounts in two studies and ultimately concluded that neither account could reliably predict behavior (Fischer, 2000). 
The current two studies investigated whether this ubiquitous effect could simply be an artifact of experimental confounds: two common experimental procedures present possible confounds that could be responsible for observed over-estimation bias. The first - the method used to control participants' body position while estimating reach - has received some consideration, but has not been resolved. The second - the connotations of the language used in experimental instructions - thus far has not been addressed as a possible driver of bias.

The first study examined the possible influence of common methods of controlling participant posture, and compared a typical, passive method of posture control with a more active method. The second study tested whether the frequent use of the word 'reach' in study instructions prompted participants to incorporate additional extension into their estimates, manifesting as the typically seen over-estimation bias. The experimental condition removed every instance of the word 'reach' in the researcher's experiment script and replaced them with the phrase "the forward extend of your arm."

\section{Study 2a: Postural Constraints}

All studies of perceived reaching ability utilize some method for controlling participant posture; in order to ensure that all participants do not suppose that they can lean forward, all participants are restrained or prompted to keep their posture upright and still throughout the study. The current study tested the effects of different methods of prompting participants to maintain proper posture during a reaching experiment. Participants were either encouraged to sit upright using a passive, tactile reminder or were actively required to maintain posture in order to keep a foam ball from falling to the floor. If actively involving participants in the task made them more aware of their bodies in space and less inclined to implicitly assume that they could lean 
forward, then we expected that participants would make more accurate estimates of their arm's reach than those in the 'passive' condition.

\section{Method}

\section{Participants}

Sixty-one University of Virginia undergraduate students participated in this study in exchange for either course credit or $\$ 5$. Two participants were excluded due to familiarity with the experimental set-up and hypotheses, leaving 59 valid participants for the analyses (43 women, 16 men).

\section{Design}

The study comprised a two-group between-subjects design (Active v. Passive postural control) examining the accuracy with which participants could estimate their arm's reach while confined to an upright posture using different methods. Reach was defined as the forward extent of the arm while the participants sat upright with shoulders parallel to the near edge of the table and their back pressed against the back of the chair. The study examined whether changing the manner in which the participant was reminded, during the study, of the necessary body position would influence their estimates.

Participants were asked to sit at the table and prompted to remain sitting straight upright by either having the apron tied around their waist and the chair, or by holding a small foam ball ( $3 \mathrm{~cm}$ in diameter) between their back and the chair back. In both conditions, the experimenter slid a small $5 \mathrm{~cm}$ diameter disc across the table both towards and away from them in counterbalanced order. Participants made their estimates by telling the experimenter to stop when the disk reached the farthest point to which they thought they could reach with their right hand. The dependent measure was calculated by the following function: 


\section{Estimated Reach $\quad=\quad$ Error Ratio Actual Reach}

A value of 1 indicated that the participant accurately estimated their own reach. A value $>1$ indicated an over-estimation of reach, while a value $<1$ indicated under-estimation.

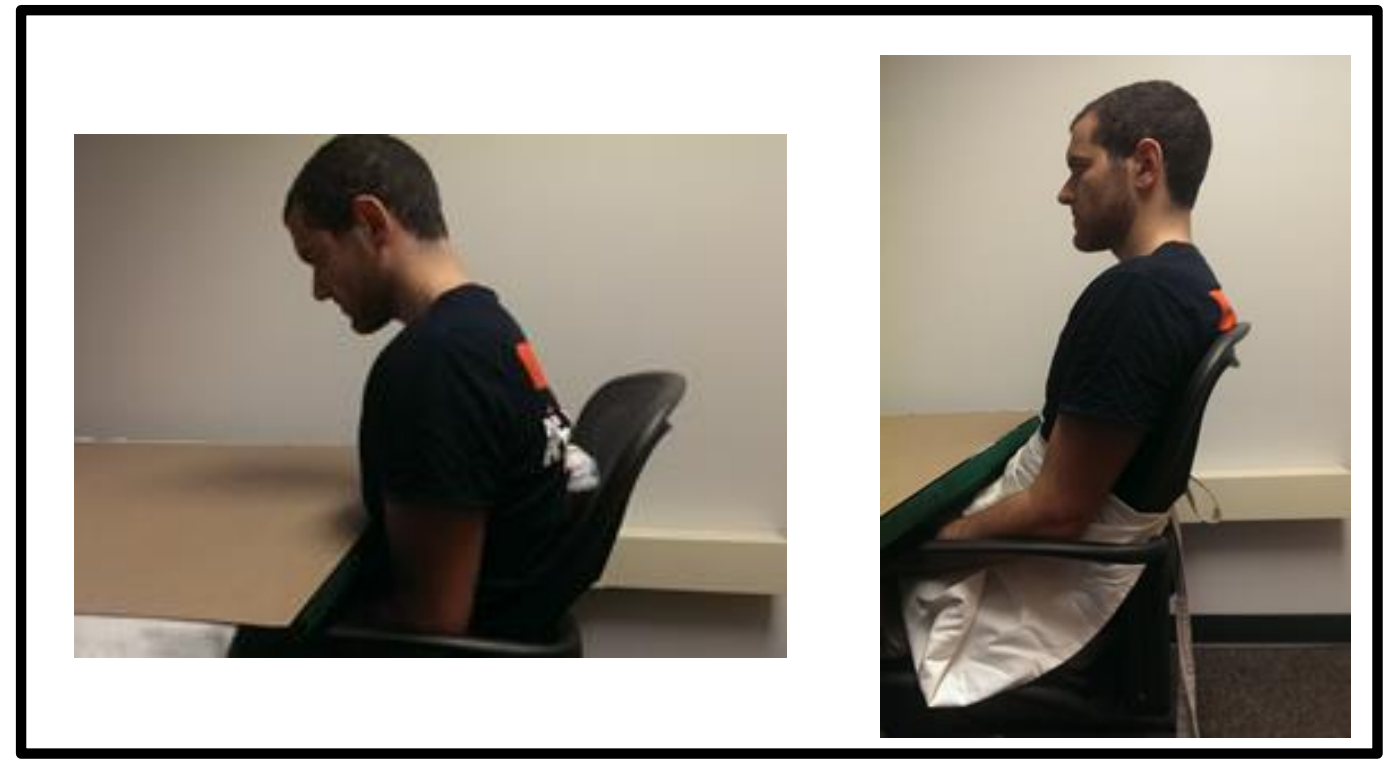

Figure 3.1. Participant displaying the active postural control condition (left) and the passive postural control condition. The participant on the left is leaning forward to make the ball visible - during the experiment, the participant would sit up straight so that their posture would match that of the participant on the right.

\section{Materials}

Participants sat at a table $75 \mathrm{~cm}$ off the ground, in a chair $45.5 \mathrm{~cm}$ off the ground. In the Passive condition, participants were prompted to keep upright posture with an apron tied around their waist and the back of the chair. In the Active condition, the experimenter placed a foam ball approximately $3 \mathrm{~cm}$ in diameter between the participant's back and the back of the chair, and pointed out that if they leaned forward at any point during the experiment the ball would fall. 
Their task was to keep the ball in place for the duration of the experiment by keeping their posture straight and upright (See Figure 3.1).

\section{Procedure}

Each participant provided informed consent, and was then asked to sit at the table. The experimenter pushed the participant's chair in so that the participant's torso was approximately 2 inches from the edge of the table. The experimenter defined 'reach' for the participant, and instructed that they keep their posture upright for the duration of the study. Depending on the condition, the participant was then either tied to the back of the chair (Passive condition) or asked to keep the foam ball from falling from behind their back (Active condition). The participant then reported their perceived reach: the experimenter slid a disc across the table, and the participant's task was to tell the experimenter to stop when the disc reached the farthest point to which the participant thought they could reach with their right hand given postural constraints. Participants in both conditions provided two estimates of their arm's reach, once as the disc was moved away from their torso across the table, and once when it was moved from the far edge of the table towards themselves. The direction of movement of the disc was counterbalanced across participants.

\section{Results: Study 2a}

An independent samples t-test compared the accuracy of participant estimates in the 'active' (M $=1.070, \mathrm{SD}=0.10)$ and 'passive' $(\mathrm{M}=1.074, \mathrm{SD}=0.12)$ conditions. Contrary to my hypothesis, results indicate no significant difference between the conditions, $\mathrm{t}(58)=0.14, p=$ .886. In both cases, one-sample t-tests indicate that participants significantly over-estimated their 
reach: active condition $\mathrm{t}(29)=3.73, p<.001$; passive condition $\mathrm{t}(28)=3.48, p=0.002$. $($ See Figure 3.2).

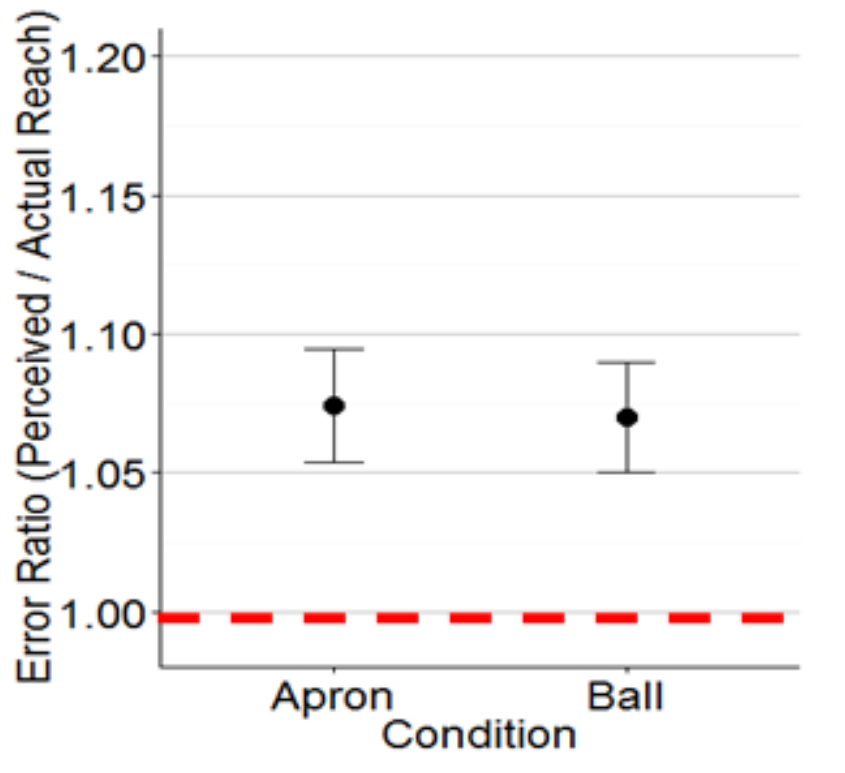

Figure 3.2. Estimate error as a function of experimental condition. Values on the y axis less than zero indicate under-estimation, while values greater than zero indicate over-estimation.

\section{Discussion: Study 2a}

Active control of one's posture does not appear to alleviate over-estimation bias.

Typically used methods of keeping participants in a controlled posture throughout a reaching/estimating task do not appear to have any influence over the accuracy of participant estimates.

\section{Study 2b: Connotation of Effortful Extension in Experiment Instructions}

This study tested the influence that the connotations of typical experimental language may have on judgments of reach. If including the word 'reach' in the instructions was priming participants to consider a more effortful extension of their bodies than the experiment called for 
explicitly, then removing the word 'reach' from the procedure should have elicited more accurate estimates of arm's reach, eliminating or reducing over-estimation bias.

\section{Method}

\section{Participants}

Thirty-three University of Virginia undergraduate students (12 men, 21 women) participated in this study in exchange for either course credit or $\$ 5$.

\section{Design}

This study employed a two-group ('reach' v. 'extent') between-subject design, examining the effect of the connotations of experimental instructions on the accuracy with which participants perceive and report their arm's reach. Data were analyzed using an independent samples t-test. In one condition, the word 'reach' was used throughout the study. In the other, the word 'reach' was replaced with 'the extent of your right arm'.

\section{Materials}

The experiment used the same table and chair as Study 2a. All participants in Study 2b were restrained using the ball and the methods from the 'active' condition in Study 2a.

\section{Procedure}

Participants were seated at a table and asked to keep their posture upright throughout the experiment. All participants were prompted to keep their posture upright for the duration of the study by holding a ball between their back and the back of the chair, as in Study2a. Participants in both conditions provided two estimates of their arm's reach as in Study 2a, but throughout the experiment the participants in the 'forward extent' condition never once heard the experimenter use the word 'reach'. Participants were shown the moving disc and asked to respond as in Experiment 2a. 


\section{Results: Study 2b}

An independent samples t-test compared the accuracy of participant estimates in the 'reach' $(\mathrm{M}=1.13, \mathrm{SD}=0.11)$ and 'forward extent' $(\mathrm{M}=1.15, \mathrm{SD}=0.14)$ conditions. Contrary to my hypothesis, results indicate no significant difference between the conditions, $\mathrm{t}(32)=0.36$, $\mathrm{p}=.721$. In both cases, participants significantly over-estimated their reach: 'reach' condition $\mathrm{t}(16)=4.99, \mathrm{p}<.001 ; \mathrm{t}(15)=4.31, \mathrm{p}<.001($ See Figure 3.2$)$

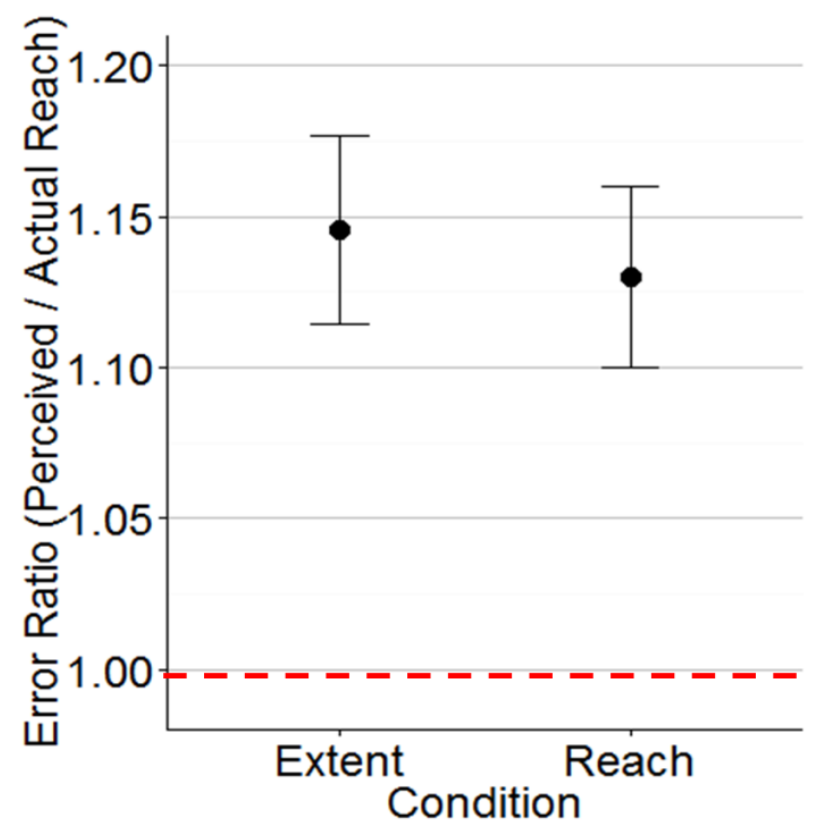

Figure 3.2. Estimate error as a function of experimental condition. Values on the y axis less than zero indicate under-estimation, while values greater than zero indicate over-estimation.

\section{Discussion: Study 2b}

Using the word 'reach' within the experiment did not influence participant estimates.

Once again, these results indicate that over-estimation bias is a robust perceptual phenomenon, 
and not simply an artifact of typical, seemingly innocuous aspects of common experimental design.

\section{GENERAL DISCUSSION}

The null findings obtained in these 2 studies suggest that over-estimation bias is real and consistent, and not caused by methods typically used in studies of perceived reaching ability. Its origins are still unresolved. 


\section{Study Set 3: Does experience influence the over-estimation bias in perceived reachability?}

\section{Introduction}

Throughout the reaching literature, researchers carefully control each participant's experience with the experimental environment both before and while they collect data. This common practice resulted from the prevailing wisdom that having any experience interacting with the experimental environment (and ostensibly having corresponding visual information) would influence participant estimates; participants would be able to use this extra information to inform and improve the accuracy of their judgments.

The developmental cognition and perception literature corroborates these expectations. Both children and adults are able to more successfully and consistently interact with their environments following task-specific experience.

Infants learn their bodies' relationship to the world through active exploration, and this learning is specific to locomotor modalities by which the child can explore independently. Proficient crawlers will not attempt to crawl over the edge of a dangerous drop, but if they are not experienced walkers and are placed on their feet and encouraged to walk, they will not hesitate to toddle over the same edge. Once those same infants become experienced walkers, they do not make the same error (Kretch \& Adolph, 2013). Furthermore, infants and toddlers' capacity to learn through experience grows along with their ability to explore independently. Young infants require more trials with feedback in order to learn how their body works in a given environment. People solidify the ability to learn quickly with experience very early in life; once infants reach 39 months of age, they will learn to avoid errors that result in falling after a single trial, just as adults will (Joh \& Adolph, 2006). 
While adults have amassed lifetimes of experiences manipulating their own relatively constant body within the environment, the body's relationship to the environment changes constantly and subtly with changing environments, goals, resources, and tasks. Adults can adeptly adjust to significant and persistent body changes: pregnant women can accurately judge the minimum passable width of a doorway even as their bellies grow, and non-pregnant controls can accommodate new girth into their perceived action boundaries with nearly the same degree of accuracy (Franchak \& Adolph, 2014). Repeated active exploration also allows adults to more accurately judge the smallest doorway through which they could walk (Franchak, van der Zalm \& Adolph, 2010). Adults can also internalize the boundaries of a new mode of locomotion with practice (in this case, moving in a wheelchair), but are only accurate at judging minimum passable height of a doorway after days of practice (Higuchi et al, 2004). The lifetime of experience each adult has accumulated must be tuned to the unique demands of each new situation, and existing work shows that continued experience in experiment-specific environments allows for this tuning.

Given the previous findings, both judgments and actions should improve with experience. Still unknown is the necessity of visual feedback during this experience. Many, if not all, previous studies have allowed participants to see either their body interacting with the environment or the pattern of optic flow associated with practiced actions. By assessing improvement absent any visual information, these proposed studies will point to the cognitive processes that support the learning and updating of action boundaries.

In the present group of studies, participants manipulated an object across a table top from directly in front of their torso all the way out to the limit of their arm's reach in several different directions. In keeping with past research, participants in one group gained this experience with 
their eyes open with the expectation that seeing the arm acting on the environment would quickly calibrate the actor to their environment and result in more accurate estimates. To test whether vision is necessary for this calibration, one group gained this experience with their eyes closed. Participants in the control condition gained no experience prior to judging their arm's reach.

\section{Study 3a: Can over-estimation bias be reduced with experience? Is vision necessary?}

\section{Purpose}

While an individual's physical limits remain relatively constant throughout time (arms do not grow and shrink from day to day), the precise limits of one's ability to act change as the actor moves through different, diverse environments. This study investigated whether contextspecific experience reduced over-estimation bias. Further, this study examined whether visual feedback of one's performance is necessary for such improvement to occur.

\section{Method: Study 3a}

65 University of Virginia students (50 women, 15 men) participated in this study in exchange for either course credit or $\$ 5$.

\section{Design}

This study comprised a one-way between subjects design with three conditions (eyes open, eyes closed, control). Prior to estimating their arm's reach over a table, participants gained experience interacting with an object on the surface of the same table. Participants slid a small bead towards and away from their body either with their eyes open or with their eyes closed. In the control condition, participants did not gain any experience, and simply estimated their arm's reach.

\section{Materials}


This study utilized the same table as Studies $2 \mathrm{a}$ and $2 \mathrm{~b}$. If participants were to repeatedly extend their arm over the same surface, it is possible that rather than learn about their body in space, all they would be learning would be landmarks and reference points on the table. To prevent participants from making their judgments based on such landmarks, the surface of the table was changed in between experience sessions, and before participants made their estimates. Two felt tablecloths of different colors (royal blue and grass green) covered the surface of the table. After the participants completed the 'experience' procedure, the top tablecloth was first rotated 90 degrees between experience and the first set of estimates, and removed to reveal the second tablecloth prior to the second set of estimates. Participants never saw their arm extended over the same surface on the table more than once per angle (See Figure 4.1).

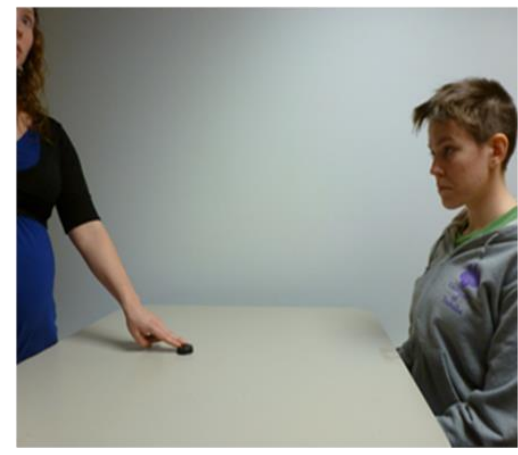

a.

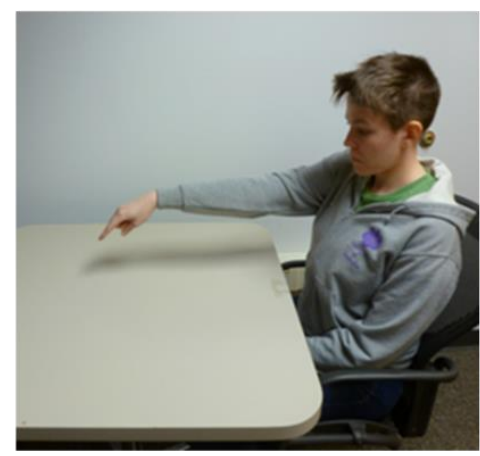

b.

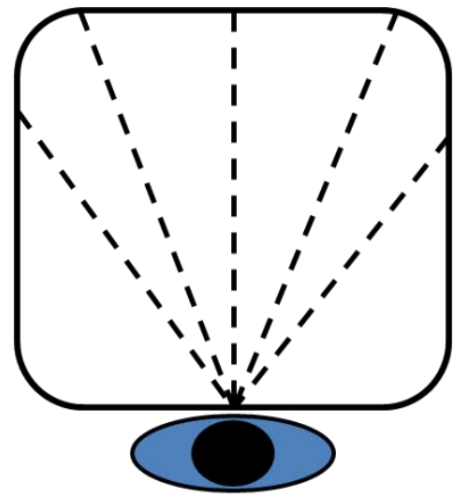

c.

Figure 4.1. In the studies within Study block 3, participants were seated at a table and asked to stop the experimenter from sliding a disc across the table when they reached their perceived action boundary (a), followed by a measure of actual reach. All of these measures were collected at all of the five angles depicted in c above. 


\section{Procedure}

After providing informed consent, each participant completed an 'experience' block where they gained experience manipulating an object at and near their action boundary on the experimental table. They then estimated their perceived reach, and finally the experimenter measured their actual reach.

\section{Experience}

While seated, the participant slid a bead across the tabletop, using their right hand, towards each of five different points on the opposite edge of the table, at five angles from the midline (30 and 15 both ipsi- and contra-lateral, and straight ahead of the participant. See Figure 6). The experimenter would then place the bead at the limit of the participant's reach and the participant would reach out and slide the bead back towards themselves. Participants either did this with their eyes open or closed. In the 'blind' condition, participants would complete the same method as in the 'sighted' condition, but with eyes closed. In the second half of the procedure, the experimenter would place the bead at the extent of the participant's reach, the participant would look at it, close their eyes, and reach out to make contact with it and draw it back towards themselves. A control group received no experience.

\section{Perceived Reach}

Next, the experimenter slid a disc across the table towards the participant, and the participant instructed the experimenter to stop when the bead reached the farthest point to which they could reach with their right hand. This was done twice from each of the five points on the table, for a total of 10 estimates per person. All distances were measured in $\mathrm{cm}$, from the point on the table that corresponded with the center of the participant's torso while seated. 


\section{Results: Study 3a}

Data were first analyzed using a mixed-effects linear model predicting the error ratio of participant estimates by condition (blind, sighted, and control) and direction $\left(30^{\circ}\right.$ and $15^{\circ}$ both ipsi and contra-lateral, and straight ahead), their interaction, and with a random effect on participant. This analysis uncovered a significant effect of direction, $\chi^{2}(4)=76.50, p<.0001$, a marginal main effect of condition, $\chi^{2}(2)=5.31, p=.07$, and no significant interaction, $\chi^{2}(8)<3$. Planned post-hoc custom contrasts were used to specifically test whether sighted ( $\mathrm{M}=1.03, \mathrm{SD}$ $=0.14)$ and blind $(\mathrm{M}=1.03,0.12)$ practice equally reduce over-estimation bias when compared to the control group $(\mathrm{M}=1.10, \mathrm{SD}=0.17)$, and found that indeed participants who received either sighted or blind experience with the experimental environment over-estimated to a significantly lesser degree than those who received no experience; $\chi^{2}(1)=5.27, p=.022$ (See Figure 4.2).

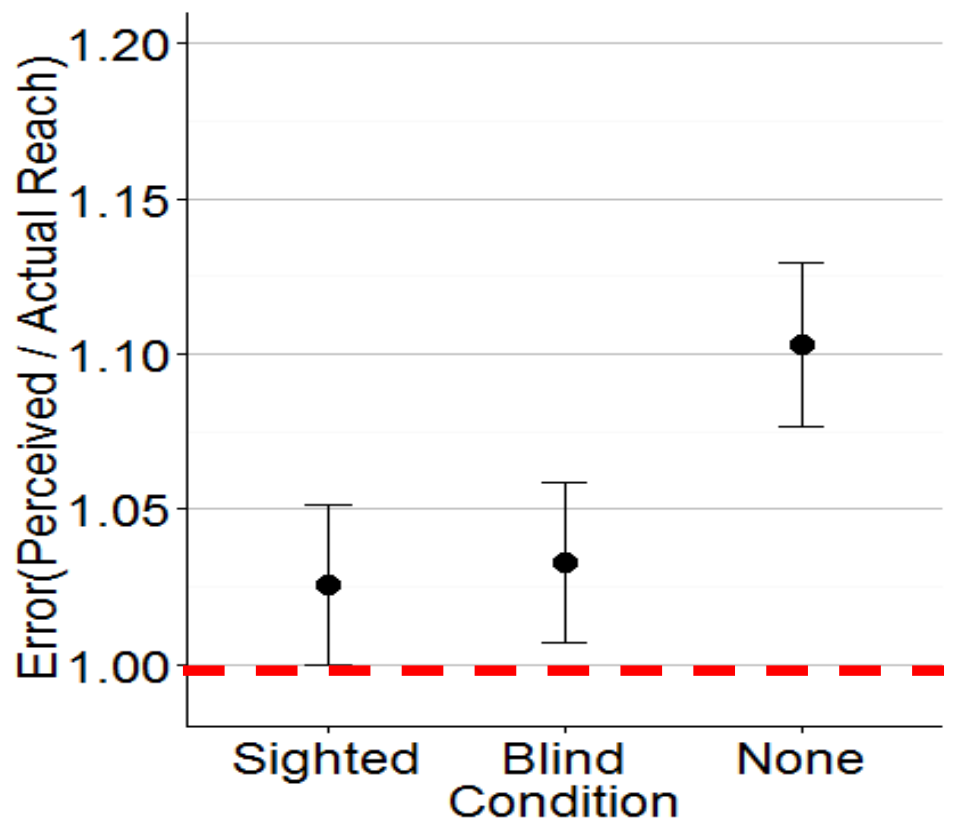

Figure 4.2. Participant estimate error as a function of Experimental condition. Error bars indicate 95\% CIs. Dotted line indicates accurate estimates. Values above 1 on the y axis indicate over-estimates. 


\section{Discussion: Study 3a}

Participants over-estimated significantly less after manipulating an object over the table, both with and without visual information about their arm's relationship to the table. These results indicate both that over-estimation bias is a malleable feature of affordance perception, and that visual feedback is not necessary to evoke such learning. Studies 2 and 3 sought to replicate this finding and remedy slight methodological issues from this study, but were unsuccessful in their ability to reproduce this finding. The studies reported in chapter 5, however, do show a consistent improvement of both judgments and actions with repeated experience, so the current studies likely did not provide participants with sufficient experience to reliably improve their judgments.

\section{Study 3b: Replication and alleviation of a confound}

This study replicated Study 3a, and altered the Experience procedure in the 'blind' condition in order to remove a small amount of visual information that may have confounded the results.

\section{Method: Study 3b}

\section{Participants}

59 University of Virginia students participated in this stud for either course credit of $\$ 5$.

\section{Design and Materials}

Study $3 b$ utilized the same experimental set-up as study $3 a$, tested the same conditions, and analyzed the data in the same way. 


\section{Procedure}

Experiment $3 \mathrm{a}$ was replicated with a minor modification. In study $3 \mathrm{a}$, during the experience portion of the 'blind' condition, participants opened their eyes to see the bead at their action boundary prior to closing their eyes and reaching out towards it. In this follow-up, participants kept eyes closed throughout the experience. To each of the 5 directions, the experimenter would indicate the correct point on the edge of the table. The participant would view this, place their right index finger on the bead, slide the bead out to the full extent of their arm, and remove their finger from the bead. After doing this towards all five directions, the experimenter would then take the bead and place it at the limit of the participant's reach while the participant kept their eyes closed. The experimenter would indicate the location of the bead by tapping on the table, and the participant would reach out towards it, place their index finger on top of it, and slide it back towards the edge of the table directly in front of their torso. Participants in both experience conditions completed this procedure to all 5 directions in pseudorandomized order, twice to each direction. Participants would cycle through each of the five directions, first sliding the bead away from their body to all five points, then sliding the bead back towards themselves, and then they would complete the whole procedure again for a total

\section{Results: Study 3b}

As in Study 3a, I first analyzed these data using a linear mixed-effects model predicting the error ratio of participant estimates by condition (blind, sighted, and control) and direction $\left(30^{\circ}\right.$ and $15^{\circ}$ both ipsi and contra-lateral, and straight ahead), their interaction, and with a random effect on participant. This analysis replicated the main effect of direction seen in the previous study, $\chi^{2}(4)=20.38, p=.0004$, but failed to replicate even a marginal effect of condition $\chi^{2}(2)<$ 3. As in Study 4.1, I again no interaction $\chi^{2}(8)<3$ was found. The same planned post-hoc 
custom contrasts from Study 4.1 were used to attempt to confirm a replication of the previous study, but found no such effect; $\chi^{2}(1)=0.33, p=.565$. Neither blind $(\mathrm{M}=1.10, \mathrm{SD}=0.17)$ nor sighted $(\mathrm{M}=1.11, \mathrm{SD}=0.09)$ experience reduced over-estimation bias compared to the control group $(\mathrm{M}=1.09, \mathrm{SD}=0.14)$. All groups significantly over-estimated their reach (see Figure $4.3)$.

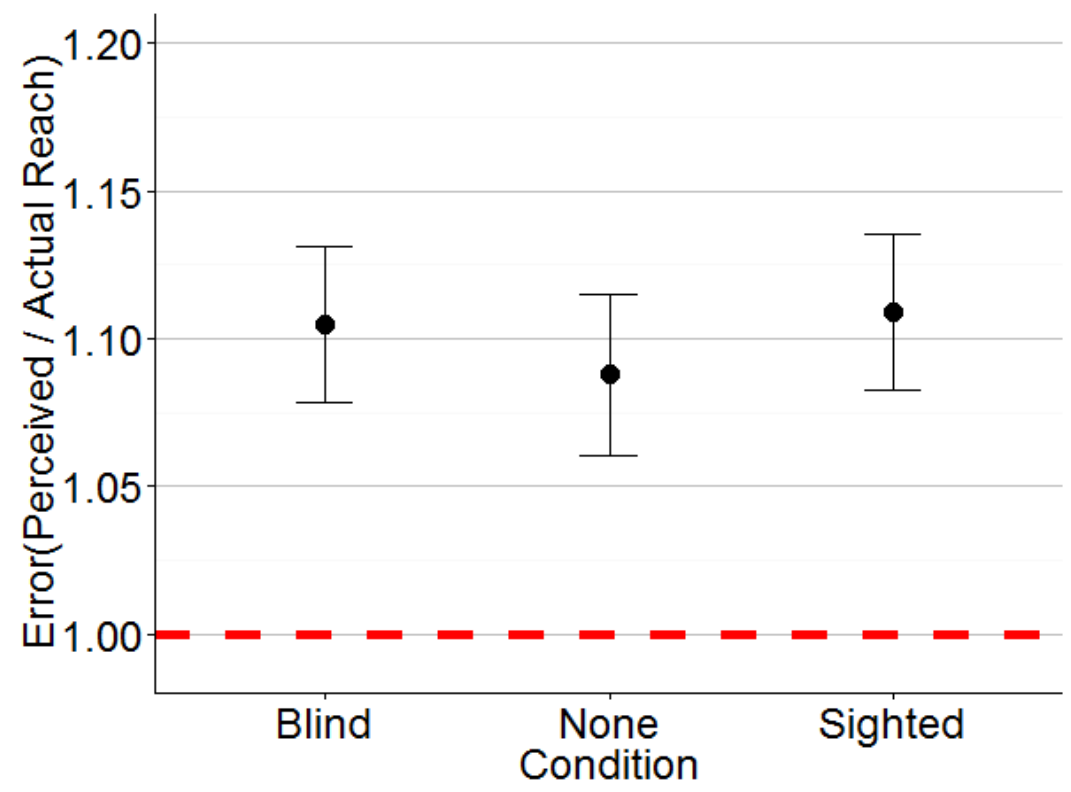

Figure 4.3. Participant error as a function of experimental condition. Error bars show $95 \%$ Cis. Dotted line indicates accurate estimates. Values above 1 on the y axis indicate

\section{Discussion: Study 3b}

Study $3 b$ did not replicate the results from Study 3a; experience did not reduce or eliminate overestimation bias in either of the groups, sighted or blind. The failure to reduce over-estimation bias in the sighted group as well as the blind group, however, does indicate that the small amount of visual information provided in the 'blind' condition of Study 1 was not enough to confound 
the results. More likely, this manipulation simply did not provide either enough experience or enough context for participants to use the information they gain to improve their estimates of their arm's reach.

\section{Study 3c: Further Replication with Awareness}

Study $3 \mathrm{c}$ attempted to replicate study $3 \mathrm{a}$ for a second time, this time by doubling the amount of experience participant gained and by modifying the experience in both conditions to make it more functional and, presumably, more engaging.

\section{Method}

\section{Participants}

Sixty-two University of Virginia undergraduate students (44 women, 18 men) participated in this study in exchange for either course credit or $\$ 5$. We began the experiment with the goal of running 60 participants (20/group) and so scheduled a number of extra participants in case of no-shows or participants who provided un-useable data. No participants were excluded from the analysis, however, so all 62 participants' data were included in the analyses.

\section{Design and Materials}

Study $3 c$ utilized the same experimental set-up as study $3 a$ and $3 b$, tested the same conditions, and analyzed the data in the same way.

\section{Procedure}

Materials and procedure were identical to those used in Experiment 3b, excepting the instructions given to the participants at the start of the study. Participants were told how they would gain experience manipulating a bead over the tabletop, and then told that they would 
subsequently be asked to estimate their arm's reach to each of the five indicated angles across the table. They were instructed to pay particular attention to where their arm was over the table so that they could use that information to make their estimates as accurate as possible. Participants were not informed of others' tendency to over-estimate reach, nor were they otherwise informed about typical or expected ways in which practice should improve their judgments.

\section{Results: Study 3c}

As for the data from Studies 3a and 3b, a mixed effects linear model predicting estimate error by direction and condition (with interaction) with a random effect of participant. As in study $3 \mathrm{~b}$, the analysis shows a significant effect of direction, $\chi^{2}(4)=13.23, p=.010$, but contrary to Study $3 \mathrm{~b}$, this model finds a significant effect of condition, $\chi^{2}(2)=7.56, p=.023$. Further investigation shows that this effect of condition does not follow the trend shown in Study $3 b$, specifically that blind $(\mathrm{M}=1.18, \mathrm{SD}=0.17)$ and sighted $(\mathrm{M}=1.09, \mathrm{SD}=0.12)$ conditions showed reduced over-estimation bias when compared to the 'no experience' condition ( $\mathrm{M}=$ $1.17, \mathrm{SD}=0.12$ ). New custom contrasts show that the confirmatory post-hoc planned custom contrasts also failed to show a replication of the effects seen in Study $4.1 ; \chi^{2}(1)=1.47, p=.226$ (see Figure 4.4). 


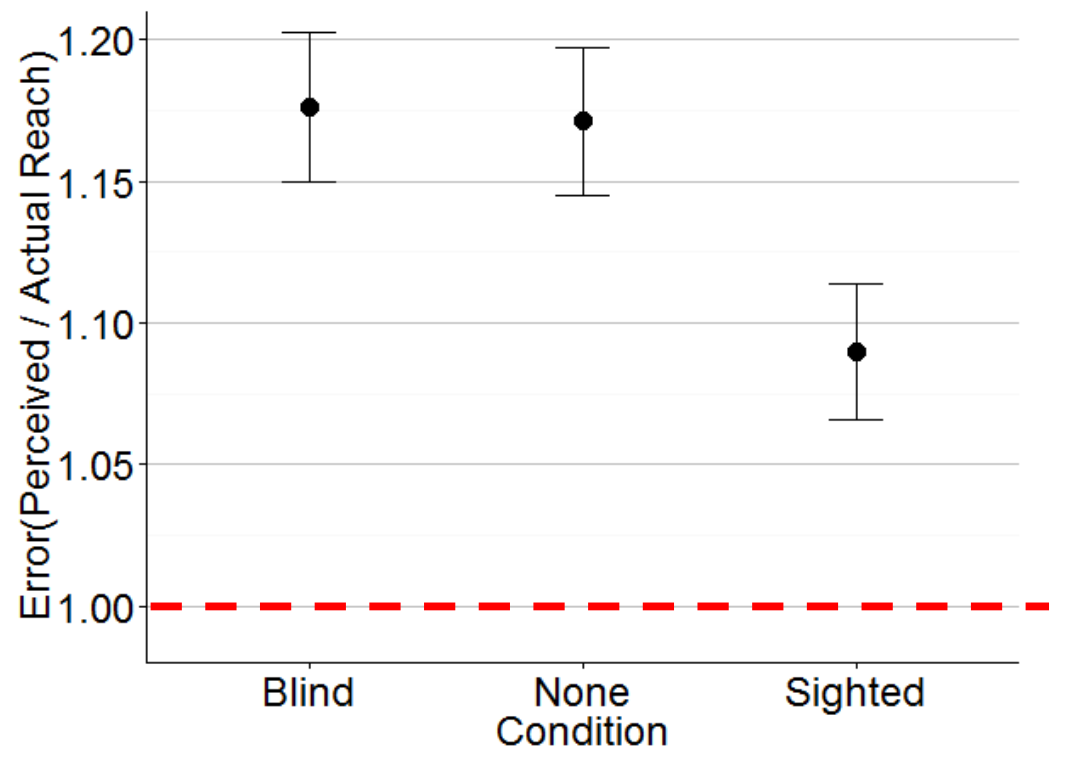

Figure 4.4. Participant error as a function of experimental condition. Error bars show $95 \%$ Cis. Dotted line indicates accurate estimates. Values above 1 on the $y$ axis indicate

\section{Discussion: Study 3c}

Like Study 3b, Study 3c did not replicate the findings from Study 3a; experience did not reduce participants' over-estimation of their arm's reach even when they were told at the outset of the experiment that they must attend closely to the position of their arm while practicing. Participants who gained experience while viewing their arm over the table did make slightly more accurate estimates of their arm's reach than those in either the blind or control conditions, but these participants still over-estimated their reach by the stereotypical $10 \%$.

\section{GENERAL DISCUSSION}

The first study in this series indicated the noteworthy findings that 1) participant bias to over-estimate their arm's reach could be reduced with task-specific experience and 2) that visual information about one's arm in relation to the environment was not necessary for such learning to occur. The second and third studies, however, do not replicate this finding. It is possible that 
this failure to replicate shows that this effect does not, in fact, exist, and that the improvement seen in the first study was an anomaly.

It is also possible that the current manipulation did not provide participants with enough experience to reliably improve their estimates. The studies detailed in Chapter 5 were undertaken with the assumption that they might detect learning, but due to the inconsistent findings in this chapter detecting a learning effect was not the primary goal of those studies. 


\section{Study Set 4: Actions are Less Biased Than Judgments, and Learning Occurs Without Vision}

\section{Introduction}

Successfully getting around in the world depends heavily and invisibly on knowing the boundaries of one's ability to act. Navigating the world seems, to most people, easy and automatic. Moving around in space, picking up objects, opening doors, and avoiding obstacles are among tasks that people can consistently do with success and without much effort or awareness. The fact that people can manage to make their way around the world without damaging property and walking into things implies that we have a relatively accurate understanding of our bodies' abilities to act - our action boundaries - and by experiencing and navigating the world we continuously both observe and experience our action boundaries in the various environments in which we conduct our lives. Given this effortless skill for successful navigation in the world, it must follow that, when asked, people can reliably report these action boundaries; by looking at the environment, an individual should be able to accurately judge and report whether or not they could fit through a doorway, climb over an obstacle, or reach an object. This information guides perception and action, so it is not unreasonable to expect that it is accessible and reportable. The literature, however, shows that this is not always the case.

The action boundary literature finds consistent and reliable errors in individuals' estimations of their own action boundaries. When shown a target and asked to make a binary yes/no judgment of its reach-ability, participants consistently over-estimated their reach (responded that they could reach targets that were too far away) when given unlimited time to report their judgment (Heft, 1993). Carello and colleagues observed participants over-estimate their arm's reach when targets were shown on a table that was both low (below waist height for 
standing participants) and at a normal height (1989). Bootsma and colleagues found that participants consistently over-estimated their ability to reach targets as they moved quickly in front of them (1992). Reachability estimates were also inflated when participants were presented with overhead targets while standing upright, and while shown targets in front of and to the side of themselves when standing on both one and two feet (Fischer, 2000). Finally, Graydon and colleagues (2013) saw all participants over-estimate their arm's reach when targets were presented at the midline or at varying angles on the side of a table contra-lateral to the arm to-bereached with, regardless of anxiety levels (although anxiety did reduce the over-estimation bias). These studies all require that participants verbally report reachability estimates, and they display a consistent over-estimation bias of around 10\% (Linkenauger et. al. 2009; Carello, et. al, 1989; Heft, 1993; Rochat \& Wraga 1997; Robinovich, 1998; Fischer, 2000; Gabbard, Cordova \& Lee, 2007; Graydon et. al, 2012). This consistent over-estimation seems at odds with our ability to navigate the world, and this inconsistency begs an explanation.

Researchers studying the perception of walkable distances grappled with a similarly persistent distortion in verbally reported estimates and developed an ingenious action-based measure to circumvent such inaccuracies. Blind walking is a measure of estimated walkable distance in which an observer views a walkable distance, closes their eyes, and then walks in a different direction until they believe they have covered the same distance as the extent they viewed (Thompson, 1983). By replacing the process of verbally reporting their estimate in units with a process of estimating using action they eliminated the previously-observed compression bias - participants shown a $10 \mathrm{~m}$ distance, for example, will report that it is $8 \mathrm{~m}$ long, but will walk the full $10 \mathrm{~m}$ when estimating with action. It is possible that reaching actions will show a similar resilience to the distortion endemic to verbally reported estimates of reaching ability; 
actions may, across the board, demonstrate more accurate assessments of distance than verballyreported estimates. Quite a bit of research has examined the accuracy of closed-loop actions, or actions that are completed with continuous visual feedback, but the current study will examine the accuracy of open-loop reaching actions which, like blind walking, are executed with either degraded or absent visual information.

In three studies, the present work explored the relationship between actions and judgments within the context of reaching. In all three studies, participants were seated at a table and shown a target at a series of distances on the tabletop in front of them. Their task was to look at the target, close their eyes, and respond in one of two ways. First, if the target appeared to be reachable, they were to reach out and touch the targets observed location on the table. Second, if the target did not seem reachable, they were to point to the location on the table where it appeared. Using this method, participant accuracy could be assessed with two measures 1) the accuracy with which participants could touch target locations within their arm's reach using a open-loop action (a non-visually guided action) and 2) the accuracy with which participants could identify beyond-reach targets as such and respond accordingly.

While the first study was undertaken as an exploratory study of reaching actions, we expected to see two trends in the results. First, we expected that actions would show resilience to over-estimation bias, just as blind walking shows resilience to compression of space found in verbal judgments. In contrast, we expected that response selection - participants' decision to touch or point at the target location - would show the typical pattern of $\sim 10 \%$ over-estimation. Studies two and three replicated the findings from Study 1, and extended both the expected and some unexpected findings. 


\section{Study 4a}

The first study was designed to establish the blind-reaching paradigm and to use it to determine whether reaching actions display the same over-estimation bias as judgments of reaching ability.

\section{Methods: Study 4a}

\section{Participants}

20 University of Virginia undergraduates participated in this study in exchange for course credit.

\section{Design}

In this within-subjects repeated measures design, participants were shown a target at one of 9 distances grouped into 4 blocks, for a total of 36 experimental trials. Participants were tasked with choosing one of two responses based on whether they perceived the target to be within reach. Data were analyzed using one of two dependent measures, depending on the question the analysis attempted to answer: the ratio of the error the participant made in their reaching motion (target distance / participant's reach response)

\section{Materials}

Locations of both participant reach and target presentation were recorded using motion tracking hardware: the Ascension trakSTAR Model 800 (8 mm OD) Sensors and Mid-Range Transmitter (Ascension Technology Corp, 2015), and labVIEW software (National Instruments, 2014). Participants sat in a chair $47 \mathrm{~cm}$ high at a table $78.5 \mathrm{~cm}$ above the ground. One motion tracking sensor was affixed to the tip of the participant's finger with athletic tape, and the other was taped to the center of a bulls-eye target mounted on cardboard.

Targets: On each of 36 trials, participants were shown a single target comprising a bullseye printed on white paper and attached to cardboard. One motion tracking sensor was fixed 
to the center of the target, and the center was marked with a dot on top of the sensor. The target was moved to one of nine locations on each trial. There were nine target distances that were centered on each participant's measured actual reach, such that the middle target (target \#5) is always shown right at the participant's fingertip. Targets were separated by $2 \mathrm{~cm}$, such that the farthest and nearest targets to the participant were separated by $16 \mathrm{~cm}$.

\section{Procedure}

To begin, participants were welcomed to the study, informed of its general contents, and asked to provide informed consent. Before being seated at the table used in the experiment, participants were instructed to keep their hands in their lap unless instructed to do otherwise. They were further instructed that throughout the study they must close their eyes prior to extending their hand and arm over the table; they could only open their eyes when their hand was resting in their lap or on the edge of the table directly in front of them. Upon sitting down at the table,

After introducing the participant to the experiment and guiding them through practice trials, the experimenter measured the extent of the participants' right arm's reach over the table. The experimenter then centered the target distances on the participants' reach so that 4 targets were shown beyond reach and 5 were within reach. On each trial, the experimenter placed the target on the table at one of 9 pre-set distances. The participant was asked to look at the target, close their eyes, reach out, and make one of two responses. If they believed that the target had appeared within reach they were to touch the table where they had seen the target. If they believed it was beyond reach, they were asked to point. Participants never saw their arm extended over the table, and never received feedback about the accuracy of their response. 
Following completion of all experimental trials, the experimenter debriefed the participant to determine whether they guessed the hypothesis of the study.

It is important to note that when participants reach out to touch a within-reach target, an under-reach, counterintuitively, indicates an over-estimation. For example, if a participant shown a target $55 \mathrm{~cm}$ away from themselves reaches out and, thinking that they are touching the target, touches the table at $50 \mathrm{~cm}$, they have under-reached by $10 \%$, or reached to only $90 \%$ of the total target distance.

\section{Results: Study $4 a$}

\section{$\underline{\text { Accuracy }}$}

Within-reach targets: A linear mixed effects model predicting reach error (target distance / participant reach response) by target distance (1-5, treated as a factor), with a random effect of participant, showed a significant effect of target distance on reach error, $\chi^{2}(4)=23.96, p<.0001$. Overall, participants display a slight bias to over-estimate their reach (to under-reach to targets), but the mean error of responses to within-reach targets was 0.985 , meaning that on average participants reached to $98.5 \%$ of the total target distance, under-reaching (over-estimating) by just $1.5 \%$. Post-hoc t-tests indicate that participants accurately responded to targets 1 , and 2 , which were the closest targets to the participant, but participants significantly under-reached towards targets 3, 4, and 5 which fell closer to the action boundary (See table 1). Overall, participants displayed a slight bias to over-estimate their reach (to under-reach to targets), but this bias did not reach the degree of the $10 \%$ over-estimation bias seen in judgments and estimates. 


\begin{tabular}{|c|c|c|c|c|}
\hline $\begin{array}{l}\text { Target } \\
\text { label }\end{array}$ & $\begin{array}{l}\text { Distance from } \\
\text { Action } \\
\text { Boundary } \\
\text { (cm) }\end{array}$ & $\begin{array}{l}\text { Mean reach Error } \\
\text { (participant reach / } \\
\text { target distance ) }\end{array}$ & $\begin{array}{l}\text { SD } \\
\text { Error }\end{array}$ & $\begin{array}{l}\text { T test comparing mean to } \\
\text { 'accurate' (one-sample t- } \\
\text { test, } \mathbf{m u}=1 \text { ) }\end{array}$ \\
\hline 1 & 8 & 0.998 & 0.072 & $\mathrm{t}(81)=-0.17, p=.863$ \\
\hline 2 & 6 & 0.992 & 0.066 & $\mathrm{t}(83)=-1.05, p=.298$ \\
\hline 3 & 4 & 0.990 & 0.060 & $\mathrm{t}(83)=-1.47, p=.146$ \\
\hline 4 & 2 & 0.977 & 0.072 & $\mathrm{t}(83)=-2.91, p=.005^{*}$ \\
\hline 5 & 0 & 0.967 & 0.062 & $\mathrm{t}(83)=-4.93, p<.0001 *$ \\
\hline
\end{tabular}

Table 1: Mean and standard deviation of reach error, along with one-sample t-tests comparing the mean error in reaches to each target to an accurate reach (1).

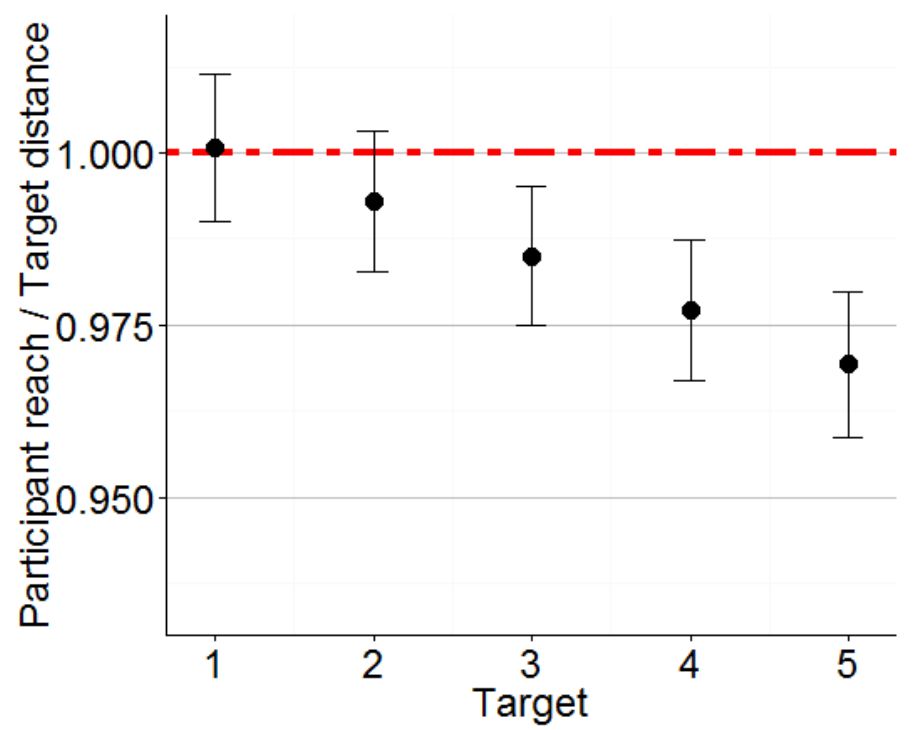

Figure 5.1. Participants' reaching error as predicted by target. Values less than 1 on the y axis indicate under-reach (overestimation). Target 1 appeared closest to the participant, while target 5 appeared right at their action boundary.

Beyond-reach targets: A mixed effects binomial logistic regression predicting the likelihood of a correctly chosen response by target (6-9, treated as a factor) was run to assess how accurately participants could identify that beyond-reach targets were, in fact, beyond reach. This analysis showed a significant main effect of target distance, $\chi^{2}(3)=46.85, p<.0001$, such that participants were much less likely to be able to correctly identify that target 6 , the target shown only $2 \mathrm{~cm}$ 
beyond reach, was beyond reach ( $18.1 \%$ of responses were correct), than target 9 , the target shown $8 \mathrm{~cm}$ beyond reach $(64.6 \%$ of responses were correct).

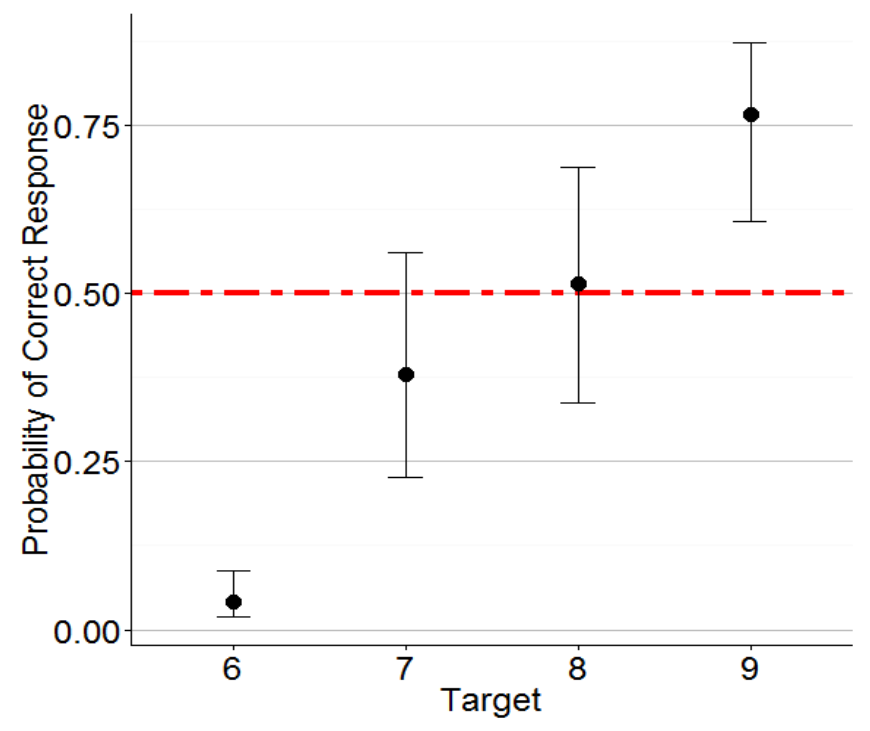

Figure 5.2: Probability of correctly responding to a beyond-reach target, as predicted by target. Target 6 appears just beyond the participants' action boundary, while target 9 is shown $8 \mathrm{~cm}$ beyond reach.

Perceived reachability of beyond-reach targets: Participants responded that they could touch many targets that were objectively beyond their reach. The error ratio of the participant reach / the target distance was calculated for each instance of an incorrect 'touch' response to a beyond reach target, and showed that participants incorrectly identified that they could reach targets that were, on average, $7 \%$ beyond their reach.

\section{Learning}

Within-reach targets: A linear mixed effects model predicting reach error (target distance / participant reach response) by experimental block was run to assess whether action accuracy improved with repeated practice. This analysis showed, unexpectedly, that participant reach 
became significantly more accurate over the course of the experiment, $\chi^{2}(3)=55.17, p<.0001$. Participants significantly under reached to all targets, on average, in the first block of trials; $(\mathrm{M}=$ $0.95, \mathrm{SD}=0.068), \mathrm{t}(103)=6.61, \mathrm{p}<.0001$, but became accurate by the fourth, final block $(\mathrm{M}=$ $1.00, \mathrm{SD}=0.064), \mathrm{t}(102)=0.035, \mathrm{p}=.972$. Interestingly, there was no significant interaction between trial block and target, indicating that the learning that occurs happened equally for all target distances $\chi^{2}(12)=4.36, p=.976$. In addition, while the mean error of participant reaches declined with experience, the variability of their performance did not. A Levene's Test of homogeneity of variance comparing the variances by experimental block indicated that none of the experimental blocks elicited significantly more varied responses than any other, $\mathrm{F}(3,313)=$ $1.60, \mathrm{p}=.188$

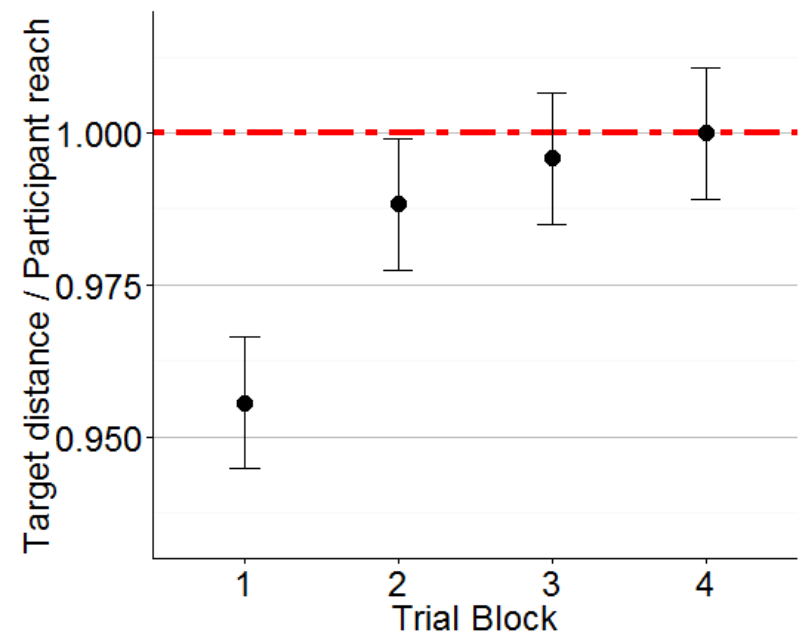

Figure 5.3: Participant reaching error as predicted by trial block.

Beyond-Reach Targets: A mixed-effects model predicting the likelihood of a correctly chosen response by target assessed whether participants became more accurate at correctly responding to beyond-reach targets over the course of the experiment. This analysis showed that yes, participants became more accurate in their response choice with practice, $\chi^{2}(3)=42.08, p<$ 
.0001 . In block 1 , participants had a $4.1 \%$ probability of correctly responding, while by block 4 they had improved to a $76.5 \%$ probability of a correct response. In addition, as seen in the within-reach learning findings above, the variability of participant responses did not decrease across blocks; according to a Levene's test, the percent of correct participant responses to beyond-reach targets did not vary significantly more or less in the first block of trials than it did in the fourth, $\mathrm{F}(1,40)=2.82, \mathrm{p}=.101$.

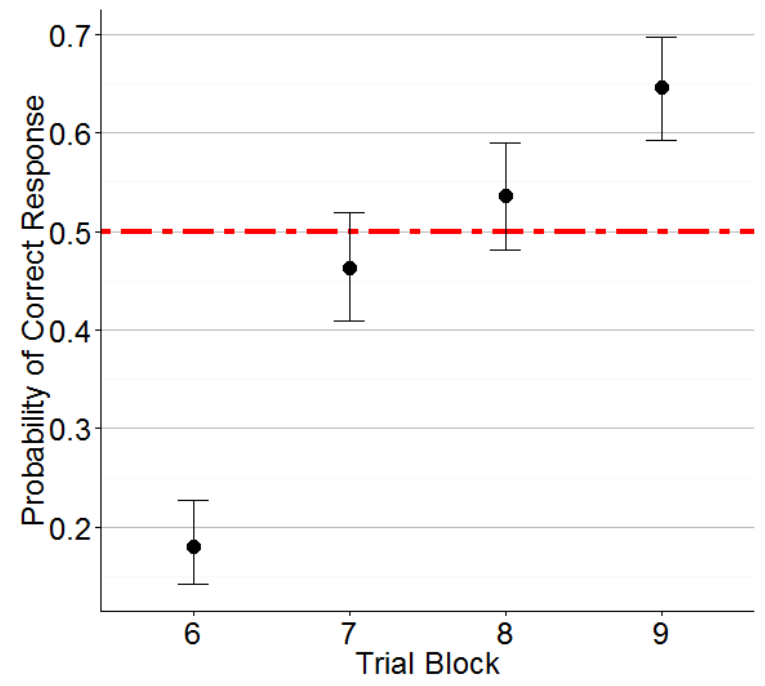

Figure 5.4: Probability of correctly responding to a beyond-reach target, as predicted by trial block.

\section{Discussion: Study 4a}

Action-selection showed approximately the same degree of over-estimation bias, $\sim 7 \%$, as verbally reported estimates of reaching ability, $10 \%$. Actions, however, did not appear to be subject to the same over-estimation bias as action selection. While they did show a slight bias when targets are closest to the action boundary (within about $4 \mathrm{~cm}$ ), this error averaged about $3 \%$, rather than the typical $\sim 10 \%$ found in both verbally reported estimates and, now, action selection. 


\section{$\underline{\text { Study } 4 b}$}

Study 4 a contained one potentially significant confound that may have biased participant response selection. Due to the observed $\sim 10 \%$ over-estimation bias, centering target distances on participants' actual reach $(\mathrm{M}=49.16 \mathrm{~cm})$, and spacing targets $2 \mathrm{~cm}$ apart, a majority of targets (targets 3-9 for most participants) were perceived as within reach. Since participants would perceive the majority of targets as reachable, the overwhelmingly common response throughout the study would be to touch the table. Participants may have, then, adopted the 'touch' response as the default and made it automatically, creating the bias to 'touch' unreachable targets seen in the above data. To control for this in Study $4 b$, the center target was shifted away from the participants' measured actual reach by $10 \%$, so that the center target appeared near the participants' perceived action boundary. If the position of the targets was creating a response bias in Study 4a, then these changes in Study $4 \mathrm{~b}$ should eliminate any results showing an overestimation bias to beyond-reach targets.

\section{Methods: Study 4b}

\section{Participants}

25 University of Virginia undergraduates participated in this study in exchange for course credit.

\section{Materials and Procedure}

Study $4 \mathrm{~b}$ was identical to Study $4 \mathrm{a}$, except that the nine targets were centered on the participant's approximate perceived reach, rather than their measured actual reach. With this standard, 3 targets appear within the participants' reach, while 6 appear beyond.

\section{Results: Study 4b}

\section{$\underline{\text { Accuracy }}$}


Within-Reach Targets: A linear mixed effects model predicting reach error (target distance / participant reach response) by target distance (1-3, treated as a factor), with a random effect of participant, showed no significant effect of target distance on reach error, $\chi^{2}(2)=3.14, p=.208$. Overall, participants displayed a slight bias to over-estimate their reach (to under-reach to targets), but the mean error of responses to within-reach targets was only 0.951 , or participants on average reached to $95 \%$ of the total target distance. Post-hoc t-tests indicate that participants significantly under-reached to all targets, $\mathrm{t}(137)=-8.60, \mathrm{p}<.0001$.

\begin{tabular}{cccc}
\hline Target & $\begin{array}{l}\text { Mean reach Error } \\
\text { (participant reach / target } \\
\text { distance ) }\end{array}$ & SD Error & $\begin{array}{l}\text { T test comparing mean to } \\
\text { 'accurate' (one-sample t-test, } \\
\text { mu = 1) }\end{array}$ \\
\hline $\mathbf{1}$ & 0.958 & .067 & $\mathrm{t}(66)=-5.04, \mathrm{p}<.0001$ \\
$\mathbf{2}$ & 0.948 & .065 & $\mathrm{t}(44)=-5.37, \mathrm{p}<.0001$ \\
$\mathbf{3}$ & 0.954 & .059 & $\mathrm{t}(13)=-2.89, \mathrm{p}=0.012$ \\
\hline
\end{tabular}

Table 2: Mean and SD of reaching errors made to within-reach targets in Study 4b, as well as one-sample t-tests comparing the mean error to each target to an accurate reach of 1 .

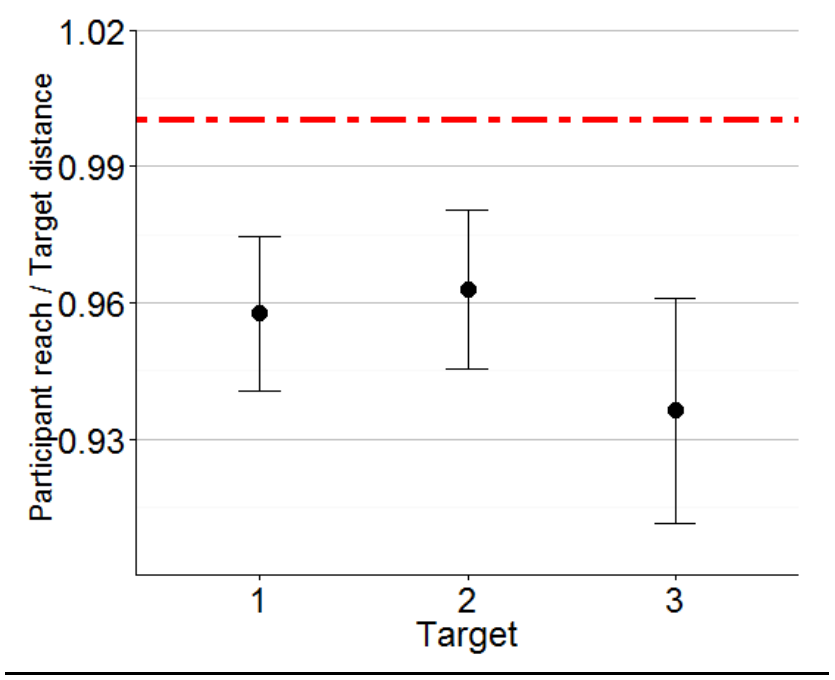

Figure 5.5: Participant reaching error as predicted by target.

Beyond-Reach Targets: A mixed effects binomial logistic regression predicting the likelihood of a correctly chosen response by target (4-9, treated as a factor) was run to assess how accurately 
participants could identify that beyond-reach targets were, in fact, beyond reach. This analysis shows a significant main effect of target distance, $\chi^{2}(5)=89.46, p<.0001$, such that participants were much less likely to be able to correctly identify that target 4 , the target shown only $2 \mathrm{~cm}$ beyond reach, was beyond reach than target 9, the target shown $12 \mathrm{~cm}$ beyond reach. The participants' probability of correctly responding to targets 4 and 5 was very low $(15.6 \%$ and $27 \%$ correct responses, respectively), just around chance to targets 6 and 7 (46.9 and 57\%) and well above chance to targets 8 and $9(73$ and $77 \%)$.

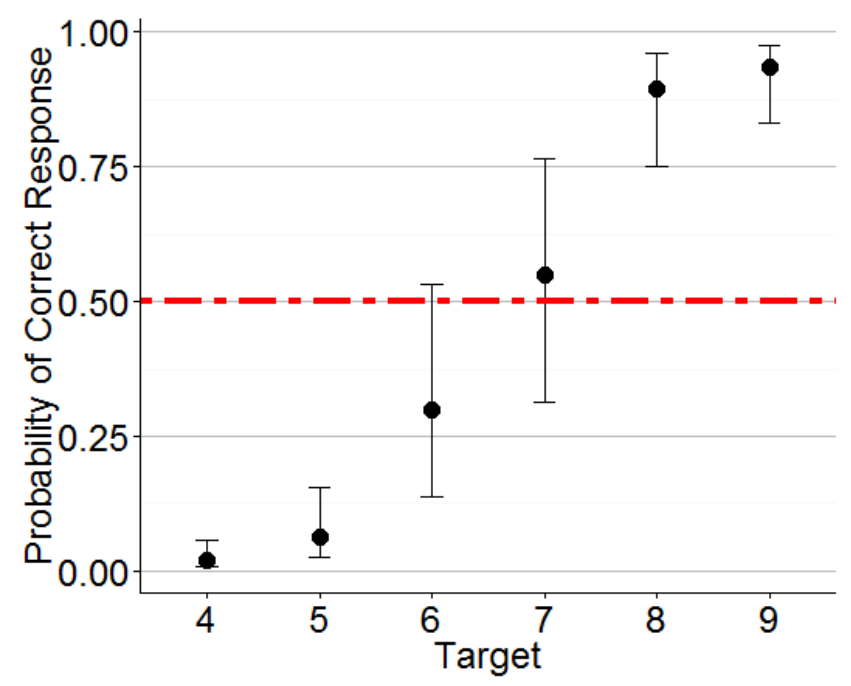

Figure 5.6: Probability of correctly responding to a beyond-reach target as predicted by target.

Perceived reachability of beyond-reach targets: Participants responded that they could touch many targets that were objectively beyond their reach. The error ratio of the participant reach / the target distance was calculated for each instance of an incorrect 'touch' response to a beyond reach target, and showed that participants incorrectly identified that they could reach targets that were, on average, $9.6 \%$ beyond their reach, nearly double the bias observed in actions towards within-reach targets. 


\section{Learning}

Within-Reach Targets: A linear mixed effects model predicting reach error (target distance / participant reach response) by experimental block was run to assess whether action accuracy improved with repeated practice. This analysis did not quite replicate the learning effect observed in Study $4 a ; \chi^{2}(3)=7.66, p=.053$. Participants did not perform more accurately during the fourth block $(\mathrm{M}=0.953, \mathrm{SD}=0.083)$ of trials than they did during the first block of trials $(\mathrm{M}$ $=0.975, \mathrm{SD}=0.0697) ; \mathrm{t}(5.9)=0.52, \mathrm{p}=.621$. These data did, however, replicate the finding that participants' responses did not become either more or less variable across the span of the experiment, $\mathrm{f}(3,42)=0.093, \mathrm{p}=.964$.

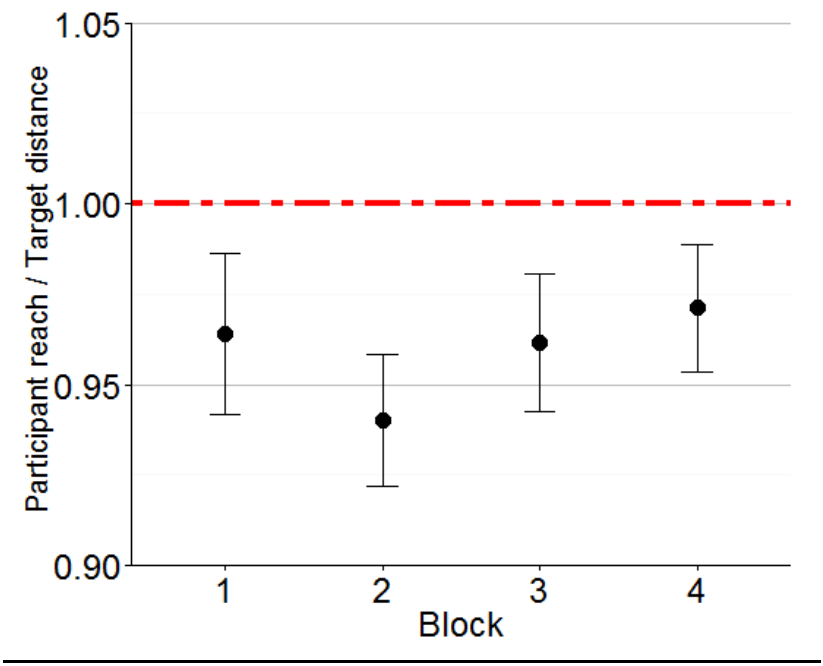

Figure 5.7: Participant reach error as predicted by experimental block.

Beyond-Reach Targets: A mixed-effects model predicting the likelihood of a correctly chosen response by target assessed whether participants became more accurate at correctly responding to beyond-reach targets over the course of the experiment. Contrary to the results of the first study, the model showed no overall improvement across the experiment, $\chi^{2}(3)=4.94, p=.176$. A posthoc test did determine, however, that participants were marginally-significantly more likely to make an accurate response in the fourth block than in the first block; $t(200)=1.903, \mathrm{p}=.057$. A 
Levene's test of homogeneity of variance also replicated the finding from Study 4a that the variability of participant response-selection error did not either increase or decrease from block 1 to block $4, \mathrm{f}(1,32)=0.016, \mathrm{p}=.900$.

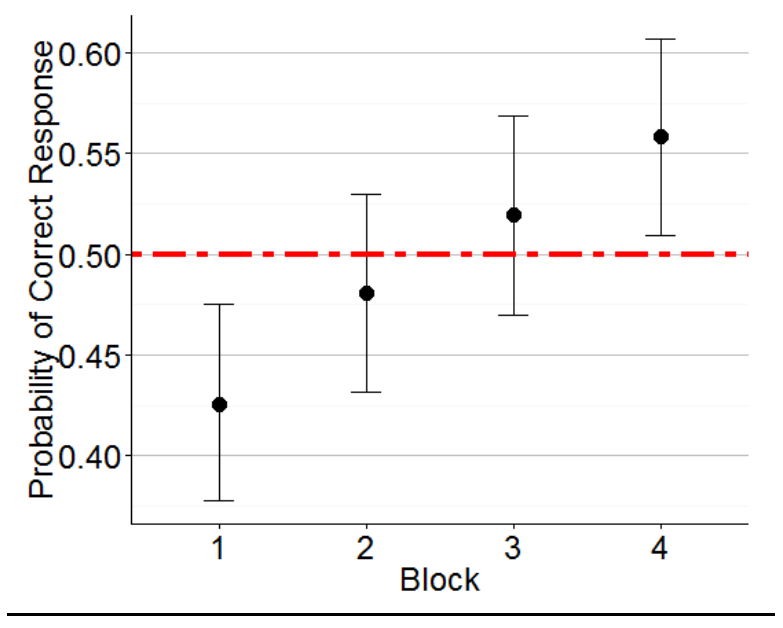

Figure 5.8: Likelihood of correctly responding to a beyond-reach target, as predicted by experimental block

\section{Discussion: Study 4b}

The data from Study $4 \mathrm{~b}$ replicated the results found in study 1 regarding accuracy.

Response-selection showed the same over-estimation bias as previously seen in verbally reported estimates, while actions showed a diminished over-estimation bias. Further, these results showed that participants in Study 4a were not biased in their response selection by the characteristics of the study, so these results are not a result of demand characteristics. These results did not, however, replicate the improvement in actions to within-reach targets seen in Study 4a.

\section{Study 4c}

Studies $4 \mathrm{a}$ and $4 \mathrm{~b}$ suggest that participants make regular and predictable errors, and Study 4a (and to a small degree, the beyond-reach data from Study $4 \mathrm{~b}$ ) showed that repetitive, nonvisual experience can reduce these errors. Study $4 \mathrm{c}$ sought to replicate these findings, determine 
whether participants are aware of their errors and examine whether this awareness might drive the learning seen across the study.

\section{Methods: Study 4c}

\section{Participants}

20 University of Virginia undergraduates participated in this study in exchange for course credit. Data from one participant were excluded from final analysis due to a technical failure during experimental trials.

\section{Materials and Procedure}

Study 4c was identical to Study 4a, with one added step per experimental trial. On each trial after the participant completes their response, if they touch the table they were then asked to indicate whether they thought they were over-reaching or under-reaching. The accuracy of these judgements (whether the participant accurately assessed whether they over or under-reached on each beyond-reach trial) was used as an additional binary dependent measure in the data analysis.

\section{Results: Study 3}

\section{Accuracy}

Within-Reach Targets:

As in Studies 4a and b, a linear mixed effects model predicting reach error (target distance / participant reach response) by target distance (1-5, treated as a factor), with a random effect of participant, showed a significant effect of target distance on reach error, $\chi^{2}(4)=13.69$, $p$ $=.008$. Overall, participants display a slight bias to over-estimate their reach (to under-reach to targets), but the mean error of responses to within-reach targets was only 0.989 , or participants on average reached out to $98.9 \%$ of the target's total distance. Post-hoc t-tests indicate that 
participants accurately responded to targets 1,2 , and 3 , but participants significantly underreached towards targets 4 , and 5 (See figure). Overall, participants displayed a slight bias to over-estimate their reach (to under-reach to targets), but this bias did not reach the degree of the $10 \%$ over-estimation bias seen in judgments and estimates.

\begin{tabular}{ccccc}
\hline $\begin{array}{c}\text { Target } \\
\text { label }\end{array}$ & $\begin{array}{l}\text { Distance from } \\
\text { Action Boundary } \\
\text { (cm) }\end{array}$ & $\begin{array}{l}\text { Mean reach Error } \\
\text { (participant reach / } \\
\text { target distance })\end{array}$ & $\begin{array}{l}\text { SD } \\
\text { Error }\end{array}$ & $\begin{array}{l}\text { T test comparing mean } \\
\text { to 'accurate' (one- } \\
\text { sample t-test, mu }=\mathbf{~ 1 )}\end{array}$ \\
\hline $\mathbf{1}$ & 8 & 1.00 & 0.101 & $\mathrm{t}(82)=0.039, \mathrm{p}=0.969$ \\
$\mathbf{2}$ & 6 & 0.992 & 0.089 & $\mathrm{t}(68)=-0.708, \mathrm{p}=.485$ \\
$\mathbf{3}$ & 4 & 0.988 & 0.075 & $\mathrm{t}(81)=-1.46, \mathrm{p}=.147$ \\
$\mathbf{4}$ & 2 & 0.983 & 0.067 & $\mathrm{t}(80)=-2.33, \mathrm{p}=0.022$ \\
$\mathbf{5}$ & 0 & 0.975 & 0.054 & $\mathrm{t}(45)=-3.13, \mathrm{p}=0.003$ \\
\hline
\end{tabular}

Table 3: Mean and standard deviation of reach error, along with one-sample t-tests comparing the mean error in reaches to each target to an accurate reach (1).

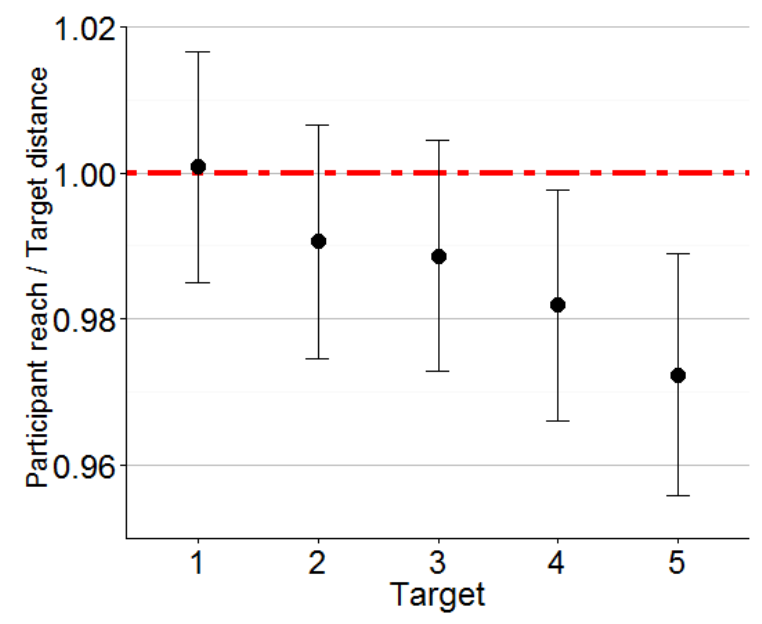

Figure 5.9: Participant reach error as predicted by target.

\section{Beyond-Reach Targets:}

A mixed effects binomial logistic regression predicting the likelihood of a correctly chosen response by target (6-9, treated as a factor) was run to assess how accurately participants could identify that beyond-reach targets were, in fact, beyond reach. This analysis showed a 
significant main effect of target distance, $\chi^{2}(3)=40.35, p<.0001$, such that participants were much less likely to be able to correctly identify that target 6 , the target shown only $2 \mathrm{~cm}$ beyond reach, was beyond reach ( $32.1 \%$ of responses were accurate), than target 9 , the target shown $8 \mathrm{~cm}$ beyond reach $(78.5 \%$ of responses were correct).

\section{Learning}

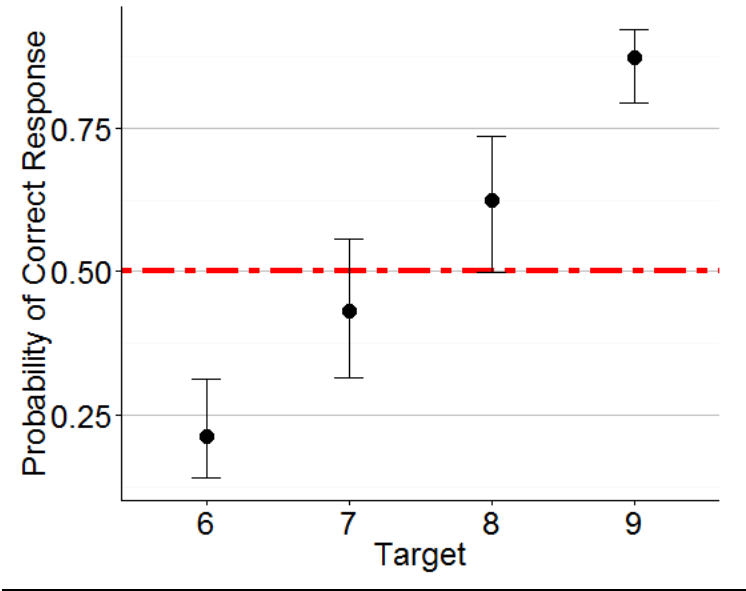

Figure 5.10: probability of correctly responding to a beyond-reach target as predicted by target.

Within-reach targets:

A linear mixed effects model predicting reach error (target distance / participant reach response) by experimental block was run to assess whether action accuracy improved with repeated practice. As in Study 4a, participants become significantly more accurate over the course of the experiment, $\chi^{2}(3)=47.47, p<.0001$. Participants significantly under reached to all targets, on average, in the first block of trials; $(\mathrm{M}=0.958, \mathrm{SD}=0.075), \mathrm{t}(86)=-5.26, \mathrm{p}<.0001$, but became accurate by the fourth, final block $(\mathrm{M}=1.00, \mathrm{SD}=0.079), \mathrm{t}(93)=0.36, \mathrm{p}=.720$. Interestingly, there was no significant interaction between trial block and target, indicating that the learning that occurs happens equally for all target distances $\chi^{2}(12)=12.7, \mathrm{p}=.391$. As seen 
in Studies 4a and 4b, a Levene's test of homogeneity of variance showed that participant responses did not become any more or less variable over the course of the experimental trials, $f(3,366)=0.104, p=.958$.

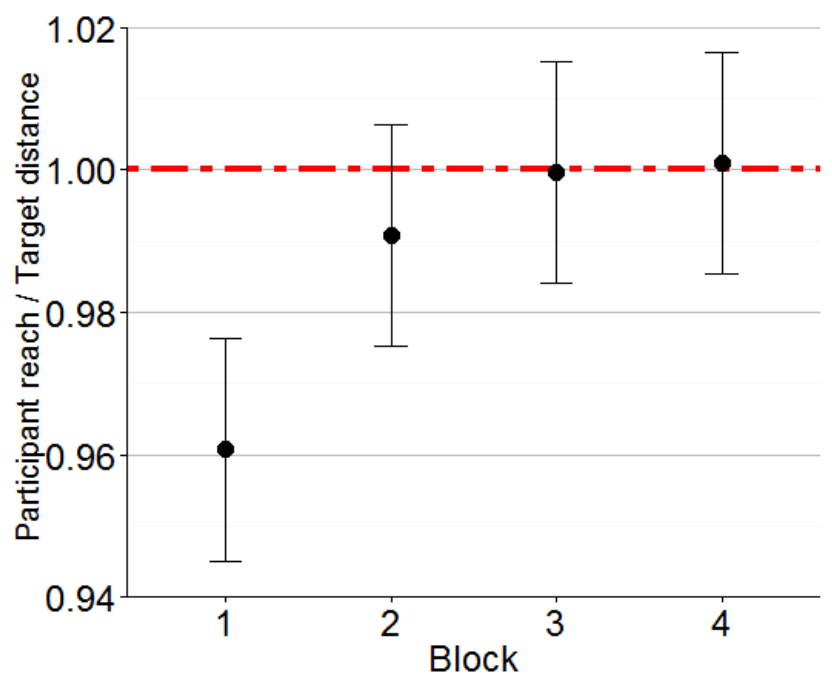

Figure 5.11: Participant reach error as predicted by experimental block.

Beyond-Reach Targets:

A mixed-effects model predicting the likelihood of a correctly chosen response by target assessed whether participants became more accurate at correctly responding to beyond-reach targets over the course of the experiment. This analysis showed that yes, participants became more accurate in their response choice with practice, $\chi^{2}(3)=17.27, p=.0006$. In block 1 , participants correctly responded to $39.2 \%$ of beyond-reach targets, while by block 4 they could correctly respond to $62.4 \%$ of targets. As seen in Studies $4 \mathrm{a}$ and b, a Levene's test of homogeneity of variance shows that participants' rates of correctly responding do not become more or less variable over the course of the experiment, $f(1,40)=0.14, p=.712$. 


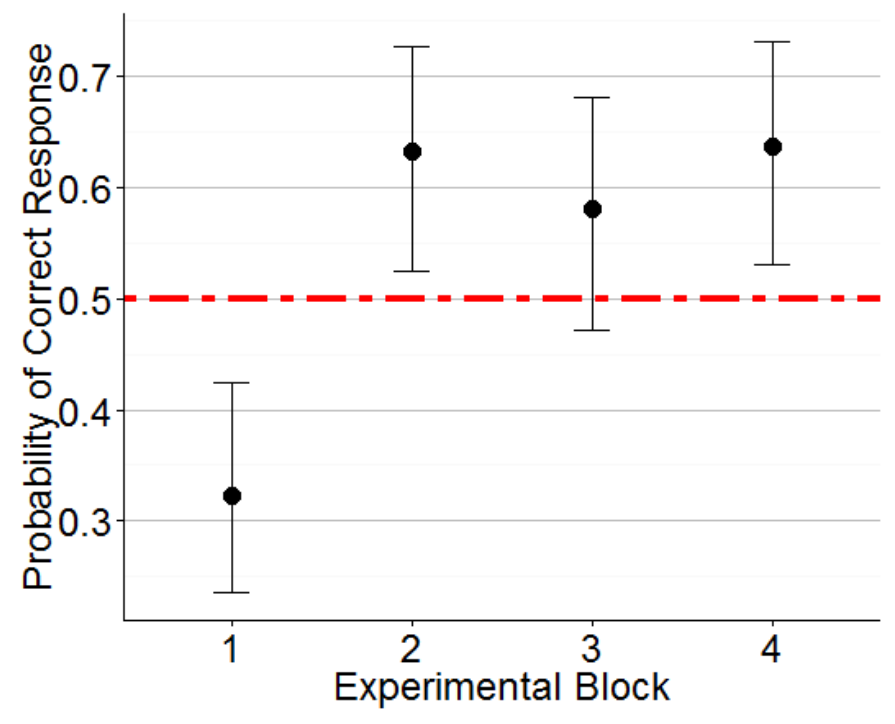

Table 5.12: The probability of correctly responding to a beyond-reach target as predicted by experimental block.

\section{$\underline{\text { Awareness of Error }}$}

Participants' awareness of their own performance could potentially explain the learning effects seen in Studies 4a and 4c. A linear model predicting improvement in actions by a score of participants' awareness of their performance was fit to assess this possibility. An improvement value was calculated for each participant by taking the mean of each participants' error scores to all targets in blocks 1 and 4, centering them on zero and taking the absolute value in order to consider 'improvement' as movement of estimates towards 'accurate' (a value of 1) either from over-estimation OR under-estimation. The awareness score used as a predictor variable was the proportion of trials on which the participant correctly judged their own accuracy in the first block of trials, with the understanding that if awareness was the driving force behind improvement, then that awareness would be most important early in the experiment. This linear model shows that greater awareness of one's errors did not significantly affect reduction in error; participants 
who are more aware of their errors did not improve to a greater degree than those who do not know how well or poorly they are doing $f(1,26)=0.104, p=.532$.(see figure 5.13 )

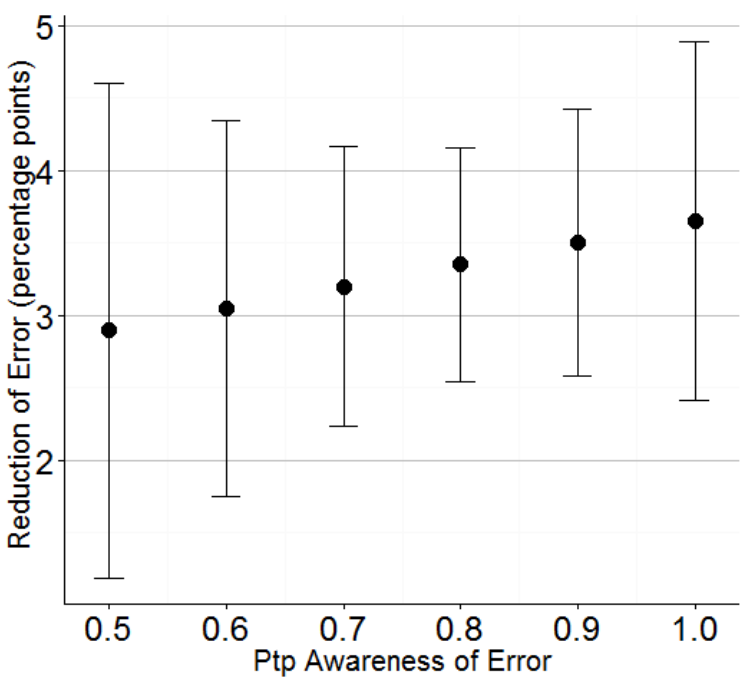

Figure 5.13: Reduction in participant error from experimental block 1 to experimental block 4 as predicted by participants' awareness of their own error during block 1 .

\section{Discussion: Study 4c}

The results from Study 4c confirmed findings and trends observed in Studies 4a and b. Actions display a small over-estimation bias when targets were near but within the action boundary, and judgments showed a stereotypical over-estimation bias when targets appeared just beyond reach. Participants were more aware of the directionality of their errors when targets were shown beyond reach, but the learning effects observed across all studies did not appear to be related to participants' ability to detect the accuracy of their actions or judgments.

\section{GENERAL DISCUSSION}

These studies produced two main findings. First, all three studies showed that reaching actions are biased, but they are far less subject to over-estimation bias than judgments of reachability. This apparent divergence between judgments and actions is consistent with at least 
three proposed theoretical generalizations, and the implications that the current findings have for all three accounts will be discussed at length in the next and final chapter.

Second, Studies $4 \mathrm{a}$ and $4 \mathrm{c}$ showed that the accuracy of both actions and judgments improves with repeated practice, but without visual, tactile or external feedback about their performance. In addition, it did not appear that participants who were more aware of their errors at the outset of the study improved to a greater extent than those who could not accurately judge their own performance. The possible sources of information that could be driving the observed learning effects will be discussed in the next chapter. 


\section{General Discussion}

\subsection{Summary of Findings}

From the present research I can draw three broad generalizations. First, over-estimation of the extent of reach does not derive from experimental confounds, but is instead a robust and consistent bias of verbally reported estimates of action boundaries. While this work does not definitively identify an underlying process or cause, its relationship to previous findings allows us to infer important characteristics of such a process.

This work also shows a partial dissociation between reaching action and judgments of reachability: actions are consistently more accurate than judgments, and while actions do display some bias that is consistent with over-estimation, it does not reach the magnitude of the bias observed both in previous work and in the judgments measured within the current studies.

Finally, I find repeated instances of participants improving the accuracy of both judgments and actions with repeated experience but without visual or performance-related feedback.

\subsection{Over-estimation Bias is not an artifact}

The studies within Chapter 3 indicate that over-estimation bias is neither driven by the manner in which participants control their posture while estimating their arm's reach, nor by the connotations of the language typically used in experimental instructions. Requiring participants to actively and consistently hold their posture upright for the duration does not prompt them to estimate their bodies' limits more accurately, as expected in Study 3.1. Likewise, the inclusion of the word 'reach' throughout a typical study examining estimated reach does not appear to drive participants to over-estimate their arms' reach. Overall, this work indicates that alternative, more conceptually complex accounts for over-estimation bias need additional pursuit. 
The first chapter of this work introduced three separate accounts for the source of overestimation bias: 1) Heft's suggestion that bias comes from post-perceptual processes (Heft, 1993), 2) the 'whole-body engagement' hypothesis that judgments of an ability necessarily involve engagement of all possibly physical degrees of freedom (Rochat \& Wraga, 1997; Robinovitch, 1998) and 3) the 'postural stability' account, which suggests that over-estimation occurs most when participants estimate from a physically stable position (Carello et. al, 1989; Gabbard, Cordova \& Lee, 2007). The current findings do not offer any contradiction to the first account, as participants were given as much time as they needed to report their estimates in all conditions. The current findings do not necessarily contradict the whole-body engagement hypothesis, as all judgments were made from a position of restricted movement. These results do indicate, however, that if this impulse to use the body's whole capacity to move when estimating ability to act does drive over-estimation bias, then it is not influenced by actively engaging the participant with the task of maintaining their posture. Finally, the postural stability account may have predicted that participants would have made more accurate estimates when they were actively engaging the muscles of their torso and required to continuously engage with their body position in the 'active' condition, which, of course, we did not observe. It is possible, however, that since participants in both conditions of that study made their estimates from a seated position, there was not enough difference in the stability of individuals' positions to find a difference in estimates. As with the first two accounts discussed above, the current results do not offer strong support for the postural stability hypothesis, nor do they refute its claims.

In summary, the studies within Chapter 3 serve two functions: first, they confirm that over-estimation bias is, in fact, a thing that happens regularly and robustly when people verbally report estimates of arm's reach. This bias is not an artifact of typically used experimental 
methods, and deserves further study. Second, it opens up the question that drove the second half of the research presented above, and validates the experimental question: are actions biased in the same way as judgments and estimates?

\subsection{Actions are less biased than judgments}

As anticipated, open-loop reaching actions displayed a much smaller over-estimation bias $(\sim 2.5 \%)$ when compared to verbally reported estimates of reachability $(9.5 \%)$. The judgments driving action selection, however, did display a stereotypical $~ 10 \%$ over-estimation bias; participants were biased to respond that they could touch targets that were regularly beyond reach. Participants did under-reach to some within-reach targets when they were closer to the action boundary, but these responses displayed a much smaller over-estimation of reach than is typically seen with either reported estimates or action selection. This outcome could suggest that while action selection judgments must be driven by a process closely related to verbally reporting perceived reaching ability, action execution relies on an at least partially distinct process.

These results are consistent with three different, somewhat disparate theoretical generalizations within perception and visually guided action literature. These three accounts for systematic trends and errors in action and perception are not necessarily mutually exclusive, and the current work, combined with possible future work, could begin to provide definitive answers to questions raised within these findings. First, Loomis and colleagues have suggested that perception feeds a single cognitive construct, and that error stems from the process of transforming the perceptual information from this construct into different kinds of output responses. Transforming perceptual information into, say, verbal output creates greater error than transforming for motor output. Second, the above results (specifically the accuracy to within- 
reach targets) are also consistent with the two-component model of target-oriented action. This two-component model account would suggest that observed under-reach to within-reach targets is an artifact of typical action patterns, and not evidence of over-estimation bias. Third and finally, the differences observed between actions and judgments mirror results that support a dual visual pathway account of visual perception, which would argue that vision informs verbal judgments and actions via two separate neural pathways.

\subsubsection{Transformation Error}

Researchers have been comparing the accuracy of explicit judgments and closed-loop actions for decades, and finding differing degrees of error in actions and judgments of distance. Loomis and colleagues found that distortion in the mapping from physical to visual space is seen in a visual matching task, but not in an open-loop motor task (Loomis, DaSilva, Fujita \& Fukusima 1992). Participants compressed walkable distances when asked to estimate them, but when blindfolded and told to walk to the end-point of a previously viewed distance, distances traversed were generally accurate. We found a similar result in our study with an open-loop motoric reaching task; the motor task shows a much smaller susceptibility to error than the verbal task of judging whether or not a target was reachable.

Loomis found a linear relationship between distances estimated using visual matching task and the blind-walking task, and suggested that this relationship indicates that both kinds of responses are made using information from a single perceptual representation. He further suggested that although visual-matching judgments and actions display different degrees of error, they both derive from a process of transforming perceptual information into response output, or 'output transformation'. Transforming information into a visual matching response, for example, creates regular systematic error to a greater degree than transforming the same information into a 
motor-based response (Loomis \&Philbeck, 2008). While the current results show a similar pattern, additional work would be required to affirm Loomis's account. Loomis established that groups, on average, make judgments and actions that follow this linear pattern over a range of walkable distances, but his approach does not address whether this pattern persists at an individual level. To confirm the output transformation account, the data would need to be evaluated participant by participant, and results would need to demonstrate that a person who under-estimates, say, a walkable distance would also under-walk to the same target. When considering the range of estimates within a given sample, the person who under-estimated the walkable distance to the greatest extent (say, they thought a $10 \mathrm{~m}$ distance was actually $7 \mathrm{~m}$ ) would also under-walk that distance to the greatest extent within the sample (walking $9.5 \mathrm{~m}$ rather than the full $10 \mathrm{~m}$ ). Across both measures the motoric measure would elicit smaller errors, but the person responsible for the greatest error would be the same in both groups, just as the most accurate person would be the most accurate across both groups. Using the methods of the current work, I would expect that a person who verbally over-estimates their reach by $15 \%$ (the higher end of the range) would under-reach to a target by maybe $4.5 \%$ (they would reach $96 \%$ of the full target distance). Results like these would indicate that in each participant, a single percept underlies all estimates of and actions within a specific modality (reaching, walking, grasping, etc).

\subsubsection{Two-component model of target-directed actions}

The target-oriented reaching literature finds that, much like the current results, reaching actions typically begin by undershooting the distance to the target. A long established model of action execution - Woodworth's two-component model (1899) - identifies two separate stages of any goal-directed action. Woodworth's two-component model notes that the first "ballistic" 
stage of a movement comprises the bulk of the movement, and positions the hand, for example, in the general location of the final goal of the action. The second stage, or the "adjustment" or "precision" stage, closes the remaining distance to the target. In closed-loop actions - actions completed with continuous feedback - movements are adjusted using visual feedback (in particular, feedback about the hand's position just before the start of the precision phase) and so appear smooth and result in minimal error (Woodworth, 1899). This research shows that the ballistic phase tends to regularly under-shoot the target, and the precision phase carries the hand the rest of the way to the target (Carlton, 1979; Chua \& Elliott, 1993; Elliott, Chua, Pollock \& Lyons, 1995; Worringham, 1999; Elliott, Lyons \& Dyson, 1997). Open-loop actions, or actions that are not updated continuously via visual feedback, do not benefit from the adjustments usually made at the start of the precision phase.

Additional, complimentary work eliminating visual feedback about performance negatively influences speed and accuracy but not overall movement trajectory: Connolly \& Goodale found that participants will be slower and less accurate both when performing a completely open-loop action (acting blind) or when they can see the target of the action but are blocked from seeing the movement of their arm (1998). Actions undertaken with clear vision of the arm (but a blocked target) were just as quick and accurate as those undertaken with full vision. In addition, in precision aiming actions, sightless aiming actions elicit errors equivalent to those made when participants are under significant pressure to execute their aiming action as quickly as possible, although this study makes no mention of improvement or learning that may have happened over the course of the experimental trials (Woodworth, 1899, as cited in Elliott, Helsen \& Chua 2001). 
Considering this literature, it is possible that the under-reach that we have been interpreting as evidence of over-estimation could, in fact, be a characteristic typical of all targetoriented reaching movements. Participants perceive accurately and would act accurately if given the visual information necessary to execute a normal 'precision phase' and complete the planned action. This reasoning could extend to suggest, then, that perceptions and their subsequent actions are accurate, barring interference or degraded information which limits the precision phase of action from correcting the errors of the initial ballistic phase. Future research could examine the trajectory, acceleration/deceleration and overall patterns of the whole target-directed movement in order to establish to what degree the precision phase occurs in open-loop actions to help determine if, in fact, the errors observed in the current research come from a diminished second phase of action. Ideally such a study would track the motion of closed-loop reaches and attempt to identify some signature of the transition between the two phases of the action, possibly a point at which the acceleration of the trajectory of the motion changes dramatically, and then compare this pattern to open-loop reaching actions. If open-loop actions consist only of the first, ballistic phase, then this signature would be absent from the data. It seems more likely, considering the learning effects also observed in the current data, that the precision phase would still occur, but either comprise a larger proportion of the whole action-duration, or follow a different type of trajectory (perhaps a path more similar to the ballistic phase than those seen in closed-loop actions).

\subsubsection{Dual-Route model of visual perception}

Finally, the current results mirror evidence for the dual-route model of visual perception. This dual route model, originally proposed by Milner and Goodale in 1992, asserts that there exist two divergent cortical pathways that drive two different kinds of visual functions: a dorsal 
pathway that connects the primary visual cortex to the motor centers of the parietal lobe and use visual information to guide actions, and a ventral pathway that connects the primary visual cortex to the inferotemporal cortex and facilitates the process of identifying and labeling seen objects (Milner \& Goodale 1992). They find a host of converging evidence that suggests that these two pathways can function independently of one another, allowing an individual who has suffered damage to the ventral pathway to correctly grasp an object that they cannot label or describe the orientation of, and a person who has a damaged dorsal pathway to perform with the opposite results. This account further holds that each of these processes utilize separate percepts, unlike Loomis' account arguing that the observed dissociation results from transforming information from a single percept into different kinds of output.

Goodale and colleagues extend this line of inquiry to examine whether actions towards objects of illusorily manipulated size would display the effects of the illusion: if the dual-route hypothesis was correct, they expected that Illusions that influence the perceived size of an
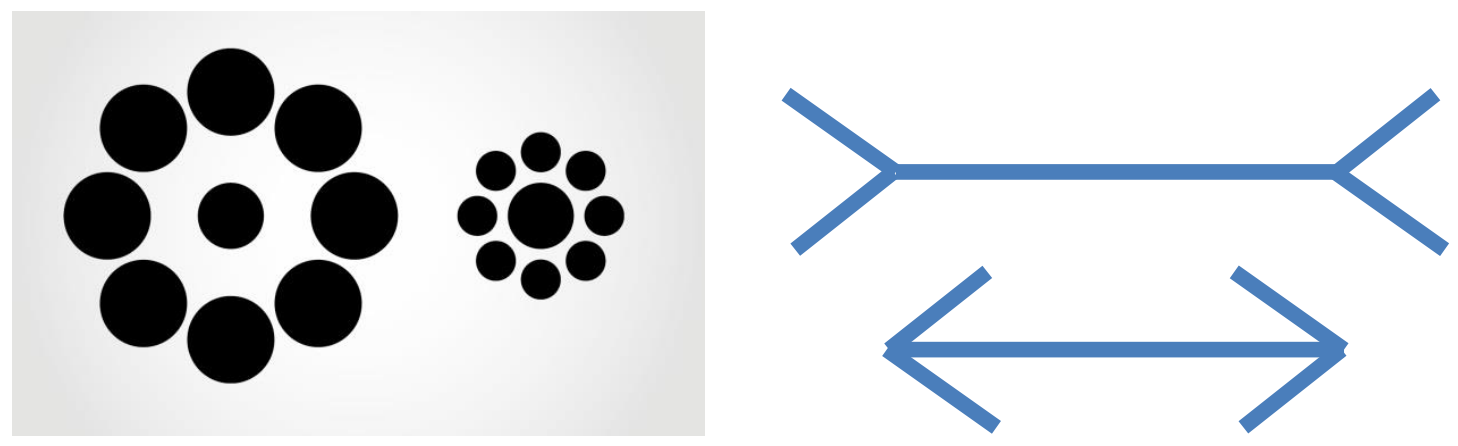

Figure 6.1: The Ebbinghause (or Tichner) illusion (left) and the Muller Lyer illusion object (the Ebbinghaus and Muller-Lyer illusions, specifically. See figure 6.1) would fail to influence the reach-to-grasp actions to the same degree as they influenced verbally reported perceptions of the object's width. That was, mostly what they found, although subsequent studies have both confirmed and failed-to-confirm this dissociation. Generally results showed 
that actions were only slightly affected by the illusion, while judgments were affected to a greater degree (Franz, Gegenfurtner, Bulthoff \& Fahle, 2000; Haffenden \& Goodale, 1998; Franz, Bulthoff \& Fahle, 2003; Gaffenden, Schiff \& Goodale, 2001; Daprati \& Gentilucci, 1997, Franz 2001). Similarly, our results show that judgments of one's capacity to act will display a different degree of error than actions themselves. In both cases, participants will err in judgments (both those that drive verbal estimates and action selection) but their actions will be much more accurate. Since Milner and Goodale's model implicates specific cortical regions in each of these processes, we could expect to be able to observe selective activity in each pathway during a modified version of the current reaching methods when the participant completed the corresponding task. Likewise, if you selectively knocked out the function of one pathway (using transcranial magnetic stimulation, for example) you would expect to see degraded performance on that pathway's corresponding task while sparing the other. Such results would offer solid support of the dual-route hypothesis while simultaneously complicating Loomis' account, which hinges on the presence of a single percept informing multiple kinds of output.

\subsection{Learning occurs over time}

Perhaps the most noteworthy of the findings presented in the previous chapters is that individuals can learn to more accurately judge their action boundaries and more accurately interact with the environment, without visual feedback about their body in space. Although plenty of works have shown that individuals can come to accurately judge their own action boundaries with experience, so far no one has shown that open-loop repeated practice can improve open-loop performance. The current work finds that repeated task-specific experience can result in more accurate actions and judgments even in the absence of visual feedback, external feedback, and tactile information about an action's endpoint. In addition, the variability 
of participant responses remains relatively constant over the course of the experiments; the improvement occurs in the mean error of participant responses. The pertinent question, now, is how does this improvement occur?

Participants receive a relatively impoverished experience of the environment throughout the experiment. They do not see their arm extended over the table, and they do not receive any feedback, either tactile or verbal from the experimenter about the accuracy or success of their actions. Participants could not rely on ostensibly the most familiar information indicating where their body was in the environment.

While the methods of the current studies deprived participants of the information that they may be more familiar with using when learning to interact with a novel environment continuous visual information, tactile information about accuracy, and external, verbal feedback about performance - participants do get feedback about their performance. In addition to having the visual information of the location of the target on the table at the start of each trial, participants gain continuous proprioceptive feedback about the movement and endpoint of their reach on each trial. Repeated trials with only this information afforded participants sufficient experience with which to improve the accuracy of both their actions and their action selection and, interestingly, their accuracy improved at about the same rate in both response modalities. Participants improved markedly from block 1 to block 2, and then either leveled off or continued to improve only gradually until the end of the experiment.

The idea that increased familiarity with a motion and how it corresponds with specific features of an environment is well documented in the perception/action literature. Participants judge the passability of a gap between two platforms more accurately when they're planning to traverse the gap using either a more familiar or more biomechanically stable action (Cole et. al., 
2013), participants will over-estimate the width of a doorway until they have experience walking through the doorway several times (Franchak et. al, 2010). All of these examples show strong evidence of participants learning their body boundaries with task-specific experience, but all also require participants to engage in fully visually-guided action. Even though a participant cannot see their body pass through a doorway as it happens, they do experience continuous optic flow coupled with the proprioceptive information gained by using their muscles to execute an action, and (if the doorway is too small) the tactile information from their arms and torso hitting the door frame. In the current study participants' performance improves using only continuous proprioceptive information about their arm's position in space and its location relative to the table (but only on responses during which they touch the table), and the visual information about where they recall having seen each trial's target relative to the whole surface of the table.

Future studies could elucidate how individuals utilize proprioceptive information when executing motor tasks, and at what point participants are able to rely on proprioceptive information to the degree that they rely on visual information. Normally-sighted individuals, when deprived of their vision while acting, can improve their performance using only proprioceptive information coupled with the visual information about where the target appeared on the table. Valuable information could be gleaned by comparing the performance of sighted individuals with highly varied levels of experience acting without visual information, and by removing the visual information from the procedure entirely. Participants could complete training to increase their experience acting without visual information in order to achieve a level of mastery of blind-action. In a study comparing experienced and novice blind actors, with aurally localized target (perhaps localized using a series of percussive clicks) it would be possible to determine the motion path, the baseline accuracy, and the capacity for improvement 
of people accustomed to relying on proprioceptive information, and those who rely primarily on vision to act accurately.

Although this new finding adds depth and nuance to the discussion of how we know what our bodies can do (and how we learn and update that information), it remains true that learning requires repetition in a specific environment. Even in the many works that have shown learning of modified physical limits or action boundaries in novel situations, both in children and adults, with visual feedback, learning happens with experience over time. Interestingly we can incorporate tools that extend our reach into our perceived action boundaries simply by imagining or anticipating using them (Witt, Proffitt \& Epstein, 2005), but still it takes significant taskspecific repetition to eliminate judgment bias. Current results, showing that improvements in performance plateau between the first and third experimental blocks, suggest that experience improves judgments somewhere between 5 and 20 trials, and the failure of the studies in Chapter 4 to find a similar learning effect with 5 repetitions corroborate this conclusion. It is now important to determine the breadth or specificity of this learning, and whether a different kind of task could enhance it.

The purpose of the action should also be taken under consideration when interpreting these results. Perhaps a more ecologically valid action would speed the learning. The task used in the studies within Chapter 4 was created with the intent of being functional, and the methods went so far as to explicitly inform participants of the action's function. Participants had to reach out and make contact with an object, and had to move an object from place to place. While the task in the studies within Chapter 5 was less functional, it was more typical to (or at least a component of) something that one might do regularly. While I expect that the number of 
repetitions drives the amount of learning that happens, the functional or familiar elements of the actions employed in the study design must be subject to continued consideration.

The current studies explored body knowledge in a very narrow, tightly controlled lab setting, although they established that learning occurs, but they were not designed to determine either the durability or the transferability of the observed newly-updated knowledge of one's action boundaries, and it remains to be seen whether some individuals can learn more successfully than others. Determining the half-life of the improvements gained from learning, understanding whether this improved body knowledge extends to tasks and environments that differ from the one in which the experience was gained, and understating what factors make some people more successful 'learners' than others would further elucidate the nature of how the body is represented within and controlled by the brain. The fact that measureable improvement in the accuracy of both actions and judgments seems driven by significant repetition indicates that inflated perceptions of reaching action boundaries (and other action boundaries that carry a low cost of making an error) are in some way advantageous, that tuning one's perceived action boundary to a specific environment is effortful, and that individuals navigate most of their lives with an inflated sense of some of the dimensions of their body in space.

\section{$\underline{6.4 .1 . ~ I m p r o v e m e n t ~ o c c u r s ~ b e l o w ~ t h e ~ l e v e l ~ o f ~ a w a r e n e s s ~}$}

The current results show marked variability in participant awareness of their accuracy, and they indicate that those participants that are more able to successfully identify the accuracy of their actions are no more likely to improve over the course of the experiment than those who are unaware of their own performance.

This outcome falls in line with previous findings from Lee, Lee, Carello \& Turvey (2012). In a study of skilled archers, Lee and colleagues examined performance accuracy over 
repeated trials without providing participants with any feedback about their performance. They found that better accuracy drove participants to perceive targets as larger, replicating an established finding (Witt \& Proffitt, 2005) in the absence of visual feedback about the end result. It is not surprising, then, that participants in the current studies could improve with limited, proprioceptive feedback, and the current work suggests that the improvement that occurs is driven by processes below the level of conscious awareness.

\subsection{In Conclusion}

You and I can make our way around our world without giving it much thought. Our bodies can do some things and cannot do others, and these abilities, along with our goals, define our relationship to the things around us. While our estimates of our action boundaries are consistently biased, our actions are much more accurate and both actions and judgments become more accurate with task-specific experience. Moreover, we now know that this improvement does not depend on visual feedback; proprioceptive feedback can fuel learning independent of on-line, continuous visual information about performance. While we have seen that perceived action boundaries are somewhat biased, this bias is consistent and small, and when tempered by the on-line visual information that typically supports actions, is accurate enough to serve successful navigation through the world. The studies reported above examine the distinction between action and perception, and show some solid evidence for at least a partial dissociation between the two processes. The current work also contains some of the first evidence that motor learning can occur without visual feedback, and does so with relatively little repetition. This work raises a whole host of new questions, some of which are new and some of which have occupied the field for years - are actions and perceptions driven by dissociated or overlapping 
processes? How would they interact? How durable is learning that happens without vision? How specific or general is it? What information, exactly, drives these improvements in performance? - but it also corroborates past findings in new and complimentary ways, and it opens up a whole new way of thinking about visually-guided action. 


\section{References}

Adolph, K. E., Bertenthal, B. I., Boker, S. M., Goldfield, E. C., \& Gibson, E. J. (1997). Learning in the development of infant locomotion. Monographs of the Society for Research in Child Development, i-162.

Adolph, K. E., Joh, A. S., \& Eppler, M. A. (2010). Infants' perception of affordances of slopes under high-and low-friction conditions. Journal of Experimental Psychology: Human Perception and Performance, 36(4), 797.

Aglioti, S., DeSouza, J. F., \& Goodale, M. A. (1995). Size-contrast illusions deceive the eye but not the hand. Current biology, 5(6), 679-685.

Bernstein, N. A. (1967). The co-ordination and regulation of movements.

Bloesch, E. K., Davoli, C. C., Roth, N., Brockmole, J. R., \& Abrams, R. A. (2012). Watch this! Observed tool use affects perceived distance. Psychonomic Bulletin \& Review, 19(2), $177-183$.

Bootsma, R. J., Bakker, F. C., van Snippenberg, F. E. J., \& Tdlohreg, C. W. (1992). The effects of anxiety on perceiving the reachability of passing objects. Ecological Psychology, 4(1), $1-16$.

Brockmole, J. R., Davoli, C. C., Abrams, R. A., \& Witt, J. K. (2013). The World Within Reach Effects of Hand Posture and Tool Use on Visual Cognition. Current Directions in Psychological Science, 22(1), 38-44.

Carello, C., Grosofsky, A., Reichel, F. D., Solomon, H. Y., \& Turvey, M. T. (1989). Visually perceiving what is reachable. Ecological psychology, 1(1), 27-54.

Canal-Bruland, R., Pijpers, J. R., \& Oudejans, R. R. (2010). The influence of anxiety on actionspecific perception. Anxiety, Stress, \& Coping, 23(3), 353-361. 
Cole, S., Balcetis, E., \& Dunning, D. (2013). Affective signals of threat increase perceived proximity. Psychological Science, 24(1), 34-40.

Davoli, C. C., Brockmole, J. R., \& Witt, J. K. (2012). Compressing perceived distance with remote tool-use: real, imagined, and remembered. Journal of Experimental Psychology: Human Perception and Performance, 38(1), 80.

Elliott, D., Helsen, W. F., \& Chua, R. (2001). A century later: Woodworth's (1899) twocomponent model of goal-directed aiming. Psychological bulletin, 127(3), 342.

Fischer, M. H. (2000). Estimating reachability: Whole body engagement or postural stability? Human movement science, 19(3), 297-318.

Franchak, J. M., van der Zalm, D. J., \& Adolph, K. E. (2010). Learning by doing: Action performance facilitates affordance perception. Vision research, 50(24), 2758-2765.

Franchak, J. M., Celano, E. C., \& Adolph, K. E. (2012). Perception of passage through openings depends on the size of the body in motion. Experimental brain research, 223(2), 301310.

Franchak, J. M., \& Adolph, K. E. (2014). Gut estimates: Pregnant women adapt to changing possibilities for squeezing through doorways. Attention, Perception, \& Psychophysics, $76(2), 460-472$.

Franz, V. H., Gegenfurtner, K. R., Bülthoff, H. H., \& Fahle, M. (2000). Grasping visual illusions: No evidence for a dissociation between perception and action. Psychological Science, 11(1), 20-25.

Franz, V. H. (2001). Action does not resist visual illusions. Trends in cognitive sciences, 5(11), 457-459. 
Franz, V. H., Bülthoff, H. H., \& Fahle, M. (2003). Grasp effects of the Ebbinghaus illusion: obstacle avoidance is not the explanation. Experimental Brain Research, 149(4), 47477.

Gabbard, C., Cordova, A., \& Lee, S. (2007). Examining the effects of postural constraints on estimating reach. Journal of Motor Behavior, 39(4), 242-246.

Gabbard, C., \& Ammar, D. (2005). Visual cues and perceived reachability. Brain and Cognition, 59(3), 287-291.

Gonzalez, C. L., Whitwell, R. L., Morrissey, B., Ganel, T., \& Goodale, M. A. (2007). Left handedness does not extend to visually guided precision grasping. Experimental Brain Research, 182(2), 275-279.

Gibson, J. (1979). The Ecological Approach to Perception. Lawrence Erlbaum Associates, Inc.

Graydon, M. M., Linkenauger, S. A., Teachman, B. A., \& Proffitt, D. R. (2012). Scared stiff: The influence of anxiety on the perception of action capabilities. Cognition \& emotion, 26(7), 1301-1315.

Haffenden, A. M., Schiff, K. C., \& Goodale, M. A. (2001). The dissociation between perception and action in the Ebbinghaus illusion: Nonillusory effects of pictorial cues on grasp. Current Biology, 11(3), 177-181.

Haffenden, A. M., \& Goodale, M. A. (1998). The effect of pictorial illusion on prehension and perception. Cognitive Neuroscience, Journal of, 10(1), 122-136.

Heft, H. (1993). A methodological note on overestimates of reaching distance: Distinguishing between perceptual and analytical judgments. Ecological Psychology, 5(3), 255-271.

Held, R., \& Hein, A. (1963). Movement-produced stimulation in the development of visually guided behavior. Journal of comparative and physiological psychology, 56(5), 872. 
Higuchi, T., Takada, H., Matsuura, Y., \& Imanaka, K. (2004). Visual estimation of spatial requirements for locomotion in novice wheelchair users. Journal of Experimental Psychology: Applied, 10(1), 55.

Ishak, S., Adolph, K. E., \& Lin, G. C. (2008). Perceiving affordances for fitting through apertures. Journal of Experimental Psychology: Human Perception and Performance, 34(6), 1501.

Joh, A. S., \& Adolph, K. E. (2006). Learning from falling. Child development, 77(1), 89-102.

Joh, A. S., Adolph, K. E., Narayanan, P. J., \& Dietz, V. A. (2007). Gauging possibilities for action based on friction underfoot. Journal of Experimental Psychology: Human Perception and Performance, 33(5), 1145.

Kretch, K. S., \& Adolph, K. E. (2013). Cliff or Step? Posture-Specific Learning at the Edge of a Drop-Off. Child Development, 84(1), 226-240

Lee, Y., Lee, S., Carello, C., \& Turvey, M. T. (2012). An archer's perceived form scales the "hitableness" of archery targets. Journal of Experimental Psychology: Human Perception and Performance, 38(5), 1125.

Libertus, K., \& Needham, A. (2010). Teach to reach: The effects of active vs. passive reaching experiences on action and perception. Vision research, 50(24), 2750-2757.

Linkenauger, S. A., Witt, J. K., \& Proffitt, D. R. (2011). Taking a hands-on approach: apparent grasping ability scales the perception of object size. Journal of Experimental Psychology: Human Perception and Performance, 37(5), 1432.

Linkenauger, S. A., Lerner, M. D., Ramenzoni, V. C., \& Proffitt, D. R. (2012). A perceptualmotor deficit predicts social and communicative impairments in individuals with autism spectrum disorders. Autism Research, 5(5), 352-362. 
Loomis, J. M., \& Philbeck, J. W. (2008). Measuring spatial perception with spatial updating and action. In Carnegie Symposium on Cognition, 2006, Pittsburgh, PA, US. Psychology Press.

Mark, L. S., Balliett, J. A., Craver, K. D., Douglas, S. D., \& Fox, T. (1990). What an actor must do in order to perceive the affordance for sitting. Ecological Psychology, 2(4), 325-366.

Milner, A. D., \& Goodale, M. A. (2008). Two visual systems re-viewed. Neuropsychologia, 46(3), 774-785.

Mishkin, M., Ungerleider, L. G., \& Macko, K. A. (1983). Object vision and spatial vision: two cortical pathways. Trends in neurosciences, 6, 414-417.

Norman, J. F., Todd, J. T., Perotti, V. J., \& Tittle, J. S. (1996). The visual perception of threedimensional length. Journal of Experimental Psychology: Human Perception and Performance, 22(1), 173.

Robinovitch, S. N. (1998). Perception of postural limits during reaching. Journal of motor behavior, 30(4), 352-358.

Rochat, P., \& Wraga, M. (1997). An account of the systematic error in judging what is reachable. Journal of Experimental Psychology: Human Perception and Performance, 23(1), 199.

Rochat, P., Goubet, N., \& Senders, S. J. (1999). To reach or not to reach? Perception of body effectivities by young infants. Infant and Child Development, 8(3), 129-148.

Thomson, J. A. (1983). Is continuous visual monitoring necessary in visually guided locomotion?. Journal of Experimental Psychology: Human Perception and Performance, $9(3), 427$ 
Warren, W. H. (1984). Perceiving affordances: visual guidance of stair climbing. Journal of experimental psychology: Human perception and performance, 10(5), 683.

Warren Jr, W. H., \& Whang, S. (1987). Visual guidance of walking through apertures: bodyscaled information for affordances. Journal of Experimental Psychology: Human Perception and Performance, 13(3), 371.

Witt, J. K., Linkenauger, S. A., Bakdash, J. Z., \& Proffitt, D. R. (2008). Putting to a bigger hole: Golf performance relates to perceived size. Psychonomic bulletin \& review, 15(3), 581585.

Witt, J. K., \& Dorsch, T. E. (2009). Kicking to bigger uprights: Field goal kicking performance influences perceived size. Perception, 38(9), 1328-1340.

Witt, J. K., \& Sugovic, M. (2010). Performance and ease influence perceived speed. Perception, 39(10), 1341-1353.

Witt, J. K., Proffitt, D. R., \& Epstein, W. (2004). Perceiving distance: A role of effort and intent. PERCEPTION-LONDON-, 33, 577-590.

Witt, J. K., Proffitt, D. R., \& Epstein, W. (2005). Tool use affects perceived distance, but only when you intend to use it. Journal of experimental psychology: Human perception and performance, $31(5), 880$. 